\title{
Neuromodulation with electrical field stimulation of dorsal root ganglion in various pain syndromes: a systematic review with focus on participant selection
}

This article was published in the following Dove Medical Press journal:

Journal of Pain Research

Ivana Vuka'

Tihana Marciuš'

Svjetlana Došenović ${ }^{2}$

Lejla Ferhatović Hamzić ${ }^{3}$

Katarina Vučić ${ }^{4}$

Damir Sapunar ${ }^{1,5, *}$

Livia Puljak ${ }^{5, *}$

'Laboratory for Pain Research, University of Split School of Medicine, 21000 Split, Croatia; ${ }^{2}$ Department of Anesthesiology, Reanimatology and Intensive Care, University Hospital Split, 21000 Split, Croatia; ${ }^{3}$ Department for Proteomics, Center for Translational and Clinical Research, University of Zagreb School of Medicine, 10000 Zagreb, Croatia; ${ }^{4}$ Department for Safety and Efficacy Assessment of Medicinal Products, Agency for Medicinal Products and Medical Devices, 10000 Zagreb,

Croatia; ${ }^{5}$ Center for Evidence-Based Medicine and Health Care, Catholic University of Croatia, 10000 Zagreb, Croatia

*These authors contributed equally to this work

Correspondence: Livia Puljak Center for Evidence-Based Medicine and Health Care, Catholic University of Croatia, llica 242, 10000 Zagreb, Croatia Email livia.puljak@unicath.hr
Objective: We conducted a systematic review about patient selection, efficacy, and safety of neuromodulation with electrical field stimulation (EFS) of dorsal root ganglion (DRG) in various painful conditions. We also analyzed conclusion statements as well as conflict of interest and financing of the included studies.

Methods: All study designs were eligible for inclusion. We searched MEDLINE, CINAHL, Embase, PsycINFO, and clinical trial registries until September 7, 2018. We assessed risk of bias by using Cochrane tool for randomized controlled trials (RCTs).

Results: Among the 29 included studies, only one was RCT, majority being case series and case reports. The evidence is based on studies with small number of participants (median: 6, range 1-152) with various painful conditions. Neuromodulation with EFS of DRG was mostly performed in participants who have failed other treatment modalities. Most of the authors of the included studies reported positive, but inconclusive, evidence regarding efficacy of neuromodulation with EFS of DRG. Meta-analysis was not possible since only one RCT was included. Conclusion: Available evidence suggest that neuromodulation with EFS of DRG may help highly selected participants with various pain syndromes, who have failed to achieve adequate pain relief with other pharmacological and nonpharmacological interventions. However, these findings should be confirmed in high-quality RCTs with sufficient numbers of participants.

Keywords: DRG, pain, neuropathic pain, chronic pain, neurostimulation, electrical stimulation

\section{Introduction}

Neurostimulation is a widely used therapeutic approach to treat various painful conditions including complex regional pain syndrome (CRPS),${ }^{1-3}$ chronic low back pain (LBP), ${ }^{4-6}$ groin pain, ${ }^{7,8}$ and pelvic pain. ${ }^{9,10}$ Neurostimulation as a therapeutic method uses electrical energy, that is, electrical field stimulation (EFS) in order to functionally activate or inhibit neuronal groups, networks, or pathways and to achieve pain relief. ${ }^{11,12}$

Primary sensory neurons and their somata in dorsal root ganglia (DRGs) are important sites where pathologic changes that lead to neuropathic pain occur, creating an opportunity for selective neuromodulation. ${ }^{13,14}$ Data from animal model studies demonstrated that neuromodulation with EFS of DRG has several advantages compared to spinal cord stimulation (SCS), allowing more precise positioning of stimulation leads with increased flexibility and reduced contact size and spacing, leading to better pain relief., ${ }^{1,13,15,16}$ Although the neuromodulation mechanism of DRG EFS has 
not been clearly elucidated, several animal studies showed that the branching point into peripheral and central process of pseudounipolar sensory neuron, that is, T-junction, ${ }^{17}$ has a filtering role in the propagation of action potentials from periphery to the spinal cord ${ }^{18}$ and can be used as a target for therapeutic stimulation that can lead to reduction of pain. ${ }^{15,17}$

Our group has recently published a systematic review about the use of neuromodulation in the context of pain from in vivo and in vitro preclinical animal model studies that showed that neuromodulation with EFS of DRGs had generally positive therapeutic effects in the context of pain. ${ }^{19}$ However, we found low methodological quality of included studies, as well a need for using standardized models and outcomes to better understand how DRG stimulation reduces pain in animal models. ${ }^{19}$ Heterogeneity of preclinical models used to study neuromodulation in the context of pain precludes any quantitative synthesis of results from different studies. ${ }^{19}$

Despite scarcity of data from preclinical models, DRG stimulation has already been used extensively in clinical settings. ${ }^{12}$ Moreover, in February 2016, the US Food and Drug Administration granted premarket approval to Axium Neurostimulator System (Spinal Modulation, Inc., Menlo Park, CA, USA, recently bought by Abbott Laboratories, Sunnyvale, CA, USA) after demonstration of its beneficial effect based on the ACCURATE study of Deer et al. ${ }^{1}$ The ACCURATE study was the only randomized controlled trial (RCT) performed in the field comparing neuromodulation with EFS of DRG with SCS for the treatment of CRPS and causalgia, with 152 participants. The study results demonstrated higher treatment success rate of DRG EFS neuromodulation compared to $\mathrm{SCS} .{ }^{1} \mathrm{CRPS}$, for which Axium Neurostimulator System was approved, is defined as chronic pain of neuropathic origin after injury of limbs such as fractures, surgery or sprains, limb immobilization or as a reflection of internal neural damage. ${ }^{20}$ Reported prevalence of CRPS is $<2 \%$ in most retrospective series. ${ }^{21}$

Several reviews about neurostimulation of DRG have been published recently. However, they mostly had a narrow focus, limited to painful condition or neuromodulation target, and they all had a number of methodological limitations. ${ }^{22,23}$ Recently published best practices on DRG stimulation by Neuromodulation Appropriateness Consensus Committee (NACC) gave a comprehensive overview of the topic, with focus on stimulation devices and procedure techniques, whereas selection of participants was mentioned briefly. ${ }^{24}$ The aim of this systematic review was to create comprehensive evidence synthesis about efficacy and safety of neuromodulation with EFS of DRG for the treatment of various painful conditions, with particular emphasis on participant selection.

\section{Methods}

\section{Study design}

We conducted a systematic review in accordance with the methods and guidelines from the Center for Reviews and Dissemination (CRD) ${ }^{25}$ and the PRISMA statement. ${ }^{26}$

\section{Protocol and registration}

The protocol of this systematic review was developed a priori and registered in the PROSPERO database (registration number: CRD42017076502).

\section{Eligibility criteria}

\section{Participants}

We included primary studies that analyzed participants with any type of pain syndrome and any intensity of pain.

\section{Interventions}

EFS of DRG, regardless of the parameters of stimulation.

\section{Comparators}

Any type of comparator was eligible. We also included studies that had analyzed EFS neuromodulation of DRG without a comparator group.

\section{Outcomes}

The main outcome measures were pain intensity and serious adverse events (SAEs) as they were defined in included manuscripts. Secondary outcome measures were any other safety data and any other pain-related outcomes. We considered all follow-up periods with no cutoff criteria.

\section{Study designs}

All study designs were eligible, including case reports. Although RCTs are considered the highest level of evidence of interventions in medicine, we were concerned that few RCTs were conducted in this field and that exclusion of nonrandomized study designs (NRSDs) would give an incomplete summary of the current evidence-base about the effects of the analyzed intervention in terms of efficacy and safety. We used the Cochrane Handbook definitions ${ }^{27}$ to determine the study design if the study design was not explicitly described in the manuscript. If a study reported cases, we considered it to be case series if it presented $>10$ participants, according to the definition of the Cochrane Handbook. We also reported study design definitions given 
by authors of included studies to present heterogeneity among definitions of NRSDs.

\section{Information sources}

We searched the following four databases: MEDLINE, CINAHL, Embase, and PsycINFO. We also searched ClinicalTrials.gov and WHO's International Clinical Trial Registry Platform to identify the ongoing studies. Databases were searched from the date of their respective inceptions and the date of the last database search was September 7, 2018, whereas for trials registry, the date of last search was October 2, 2018.

References and citations of the included studies and any potentially relevant reviews were analyzed in order to find additional eligible studies that may not have been retrieved by the database search.

\section{Search strategy}

A computer-based search strategy was designed and conducted with the assistance of an expert medical librarian, who also peer-reviewed the final version of the search strategy. Search strategy for MEDLINE via OVID (Table S1) was developed first and adapted for other databases subsequently. Studies published in any language were considered. Searches were conducted separately in each database, and subsequently the records were exported to EndNote X5 citation software (Clarivate Analytics, Boston, MA, USA). Duplicates were removed, first by software and then manually. Reference lists of all the included studies were searched. Citations and references of the included studies were downloaded from Web of Science.

\section{Study selection}

Two authors independently screened titles and abstracts of the bibliographic records retrieved via the database search (SD and LFH). Two authors also independently screened full-text manuscripts of potentially relevant studies (IV and TM). In each step, disagreements were resolved via discussion or involvement of a third author (DS). When involvement of a third author was deemed necessary, the third author would suggest the solution, with arguments, and this was in all cases accepted by the co-authors.

\section{Data collection process and data items}

A data collection form was developed for this study and piloted using five included studies and subsequently revised the form as appropriate. Two authors (IV and TM) independently extracted data in duplicate. The following data were extracted: name of the first author, year of publication, study design, intervention and comparator, number of participants, participant characteristics including inclusion and exclusion criteria, baseline characteristics, previous therapy and painful condition, follow-up period, parameters of stimulation and stimulator used, position of leads, studied outcomes, and study results regarding efficacy and safety of intervention.

\section{Risk of bias (RoB) assessment}

To assess RoB in the included studies, we used the Cochrane RoB tool for RCTs, which has seven domains, addressing bias related to random sequence generation, allocation concealment, blinding of participants and personnel, blinding of outcome assessors, incomplete outcome data, selective reporting, and other potential sources of bias. For each domain, we reported our judgment, that is, whether the risk was low, high, or unclear, and we provided a supporting comment, which explained our judgment. RoB was assessed per domain level; we did not assess RoB on outcome level, and we did not assess overall RoB on an entire study level. RoB assessment was included in our narrative analysis and conclusions.

We aimed to use the Risk of Bias in Non-randomized Studies of Interventions (ROBINS-I) tool for cohort studies, but we did not have such studies in our sample. ${ }^{28}$ Two authors independently analyzed RoB (KV and SD), and discrepancies were resolved by the third author (LP).

\section{Synthesis of results}

We grouped the results according to painful condition treated with DRG stimulation. Studies that included participants with multiple etiologies were grouped into the painful condition category with highest number of participants and mentioned in other categories if relevant. The results are presented in a narrative and tabular form. We planned to conduct meta-analysis of outcomes from RCTs, but meta-analysis was not possible since only one RCT was included. In addition, we analyzed reporting of conclusion statements for efficacy and safety in manuscript abstracts. We extracted verbatim those conclusion statements and divided them into five categories: positive conclusive, positive inconclusive, negative conclusive, negative inconclusive, and not reported. We categorized as inconclusive conclusion statements that used conditional wording about the efficacy or safety and/or indicated that more evidence is needed.

\section{Results}

Electronic databases searches yielded 2,811 records. An additional 1,473 studies were identified through supplementary search of references, citations, and other reviews. After identifying and removing duplicate records, 2,133 unique 
records remained for eligibility determination and inclusion. We analyzed a total of 39 manuscripts in full text, excluded 10 of them, and finally included 29 studies in narrative synthesis. A flowchart is presented in Figure 1, whereas characteristics of included studies are described in Tables $1-3$. Studies excluded from further qualitative synthesis $(\mathrm{N}=10)$ and reasons for their exclusion are presented in Table S2. Ongoing studies found in clinical trial registries are presented in Table S3.

Included studies had various study designs: 1 RCT, 8 before and after comparisons, 2 case series, and 18 case reports. Liem et al $(2013,2015)$ reported the results of the same study with data shown for 6 months $^{3}$ and 12 months follow-up time periods, ${ }^{2}$ so we considered both of them. Two studies from van Velsen et al analyzed the same patient; therefore, we left both the references but included it in analysis only once. ${ }^{29,30}$ Due to a large number of case reports and case series, overall, the included studies were with very small median number of participants 6 (range: 1-152). Only one included RCT and two observational studies had higher number of participants.

\section{Pain syndromes analyzed in included studies}

Included studies analyzed the following painful conditions: CRPS, ${ }^{1-3,31-36}$ LBP, ${ }^{5,6,37-40}$ groin pain, ${ }^{7,8,41}$ pelvic girdle pain, ${ }^{9,10}$ peripheral neuropathy, ${ }^{29,30,42}$ peripheral diabetic neuropathy, ${ }^{43}$ phantom limb pain, ${ }^{44}$ chronic intractable pain in the coccyx, ${ }^{45}$ chronic testicular pain, ${ }^{46}$ anterior cutaneous nerve entrapment syndrome (ACNES), ${ }^{47}$ loin pain hematuria syndrome (LPHS). ${ }^{48}$

\section{Studies awaiting classification}

No results have yet been published for several completed clinical trials retrieved via search of clinical trials registers. One study was classified as completed with no results (NCT02169401). The trial authors informed us that the manuscript has been submitted. Other studies were classi-

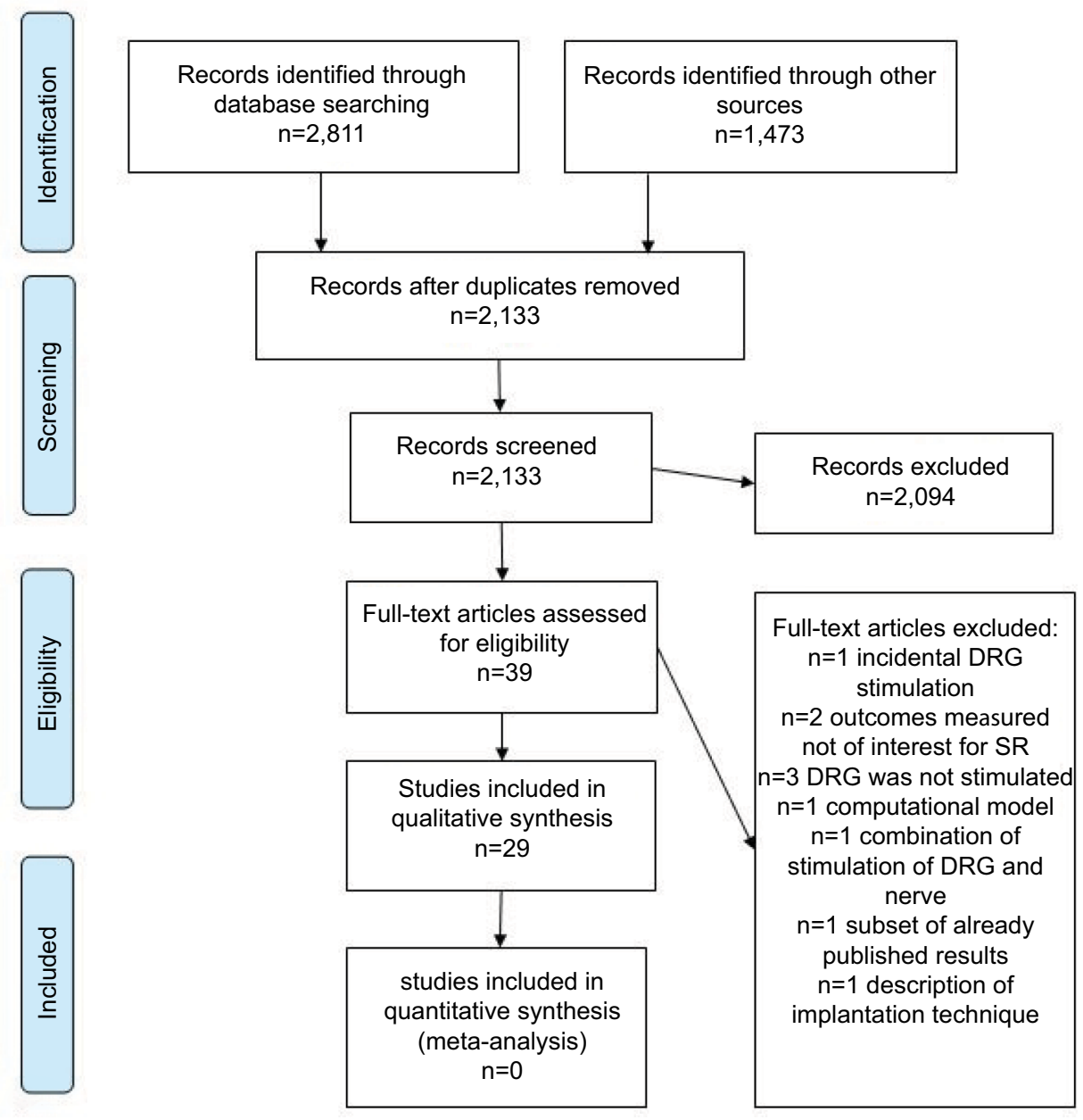

Figure I Study flowchart.

Abbreviations: DRG, dorsal root ganglion; SR, systematic review. 


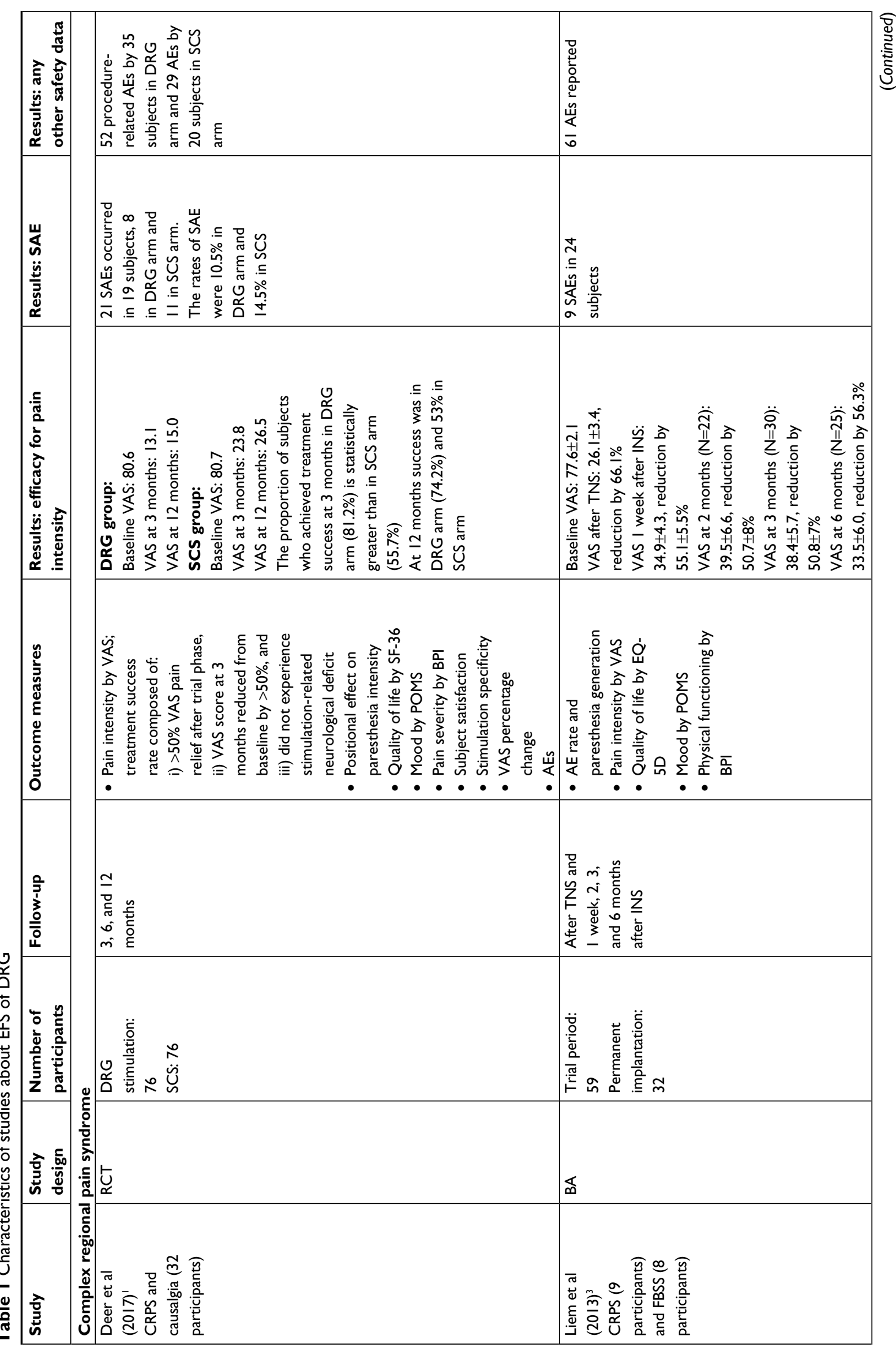




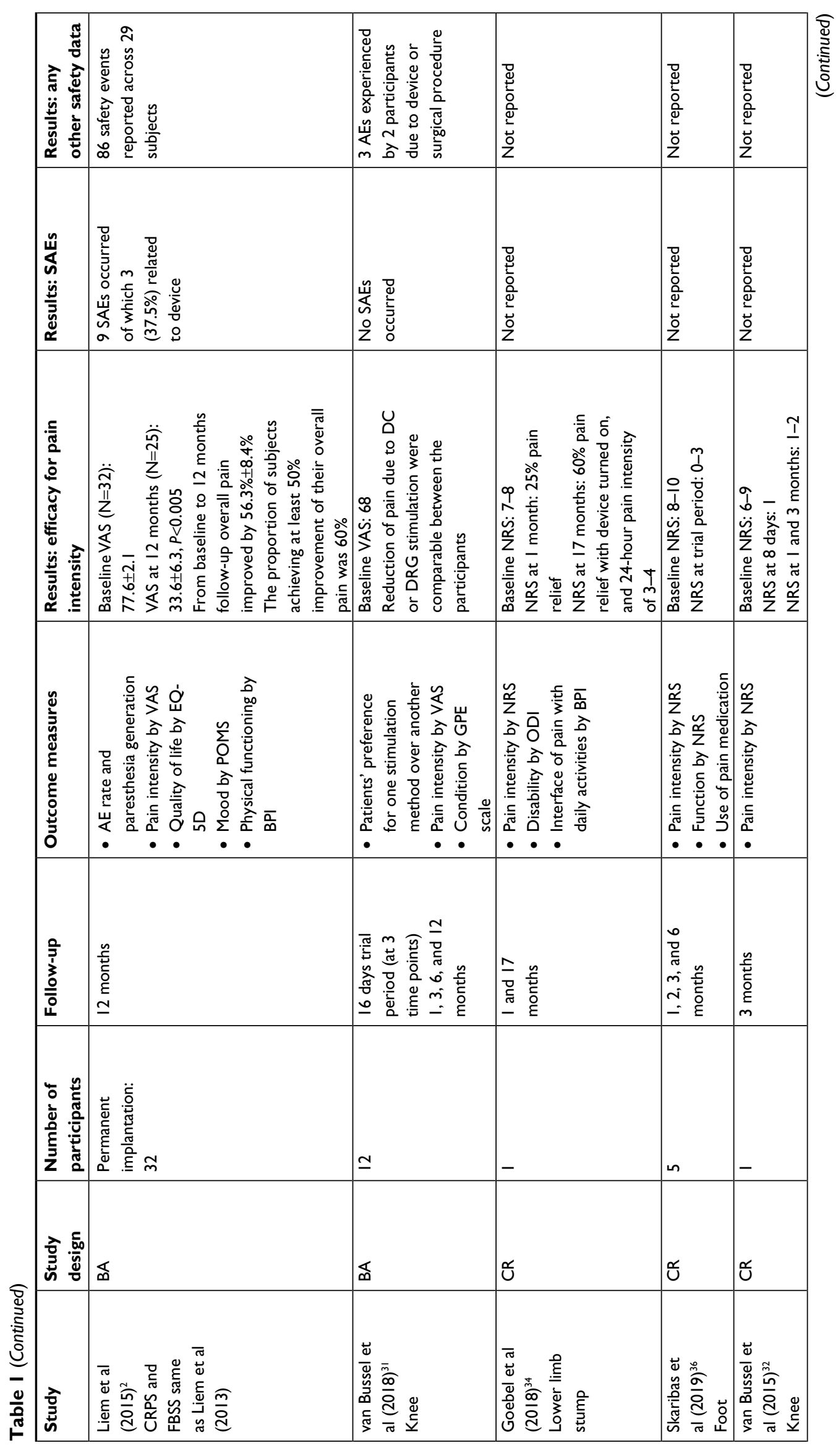




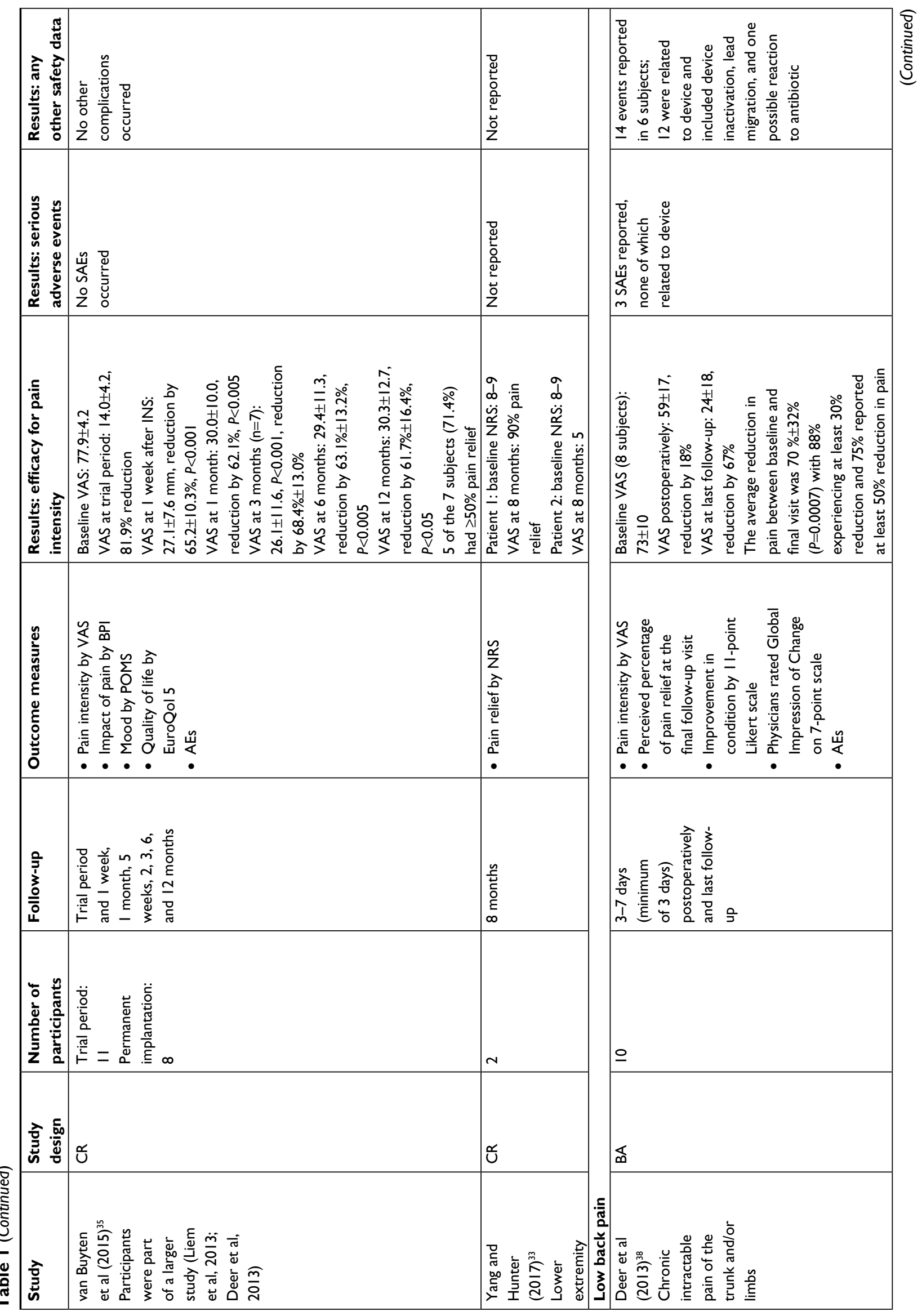




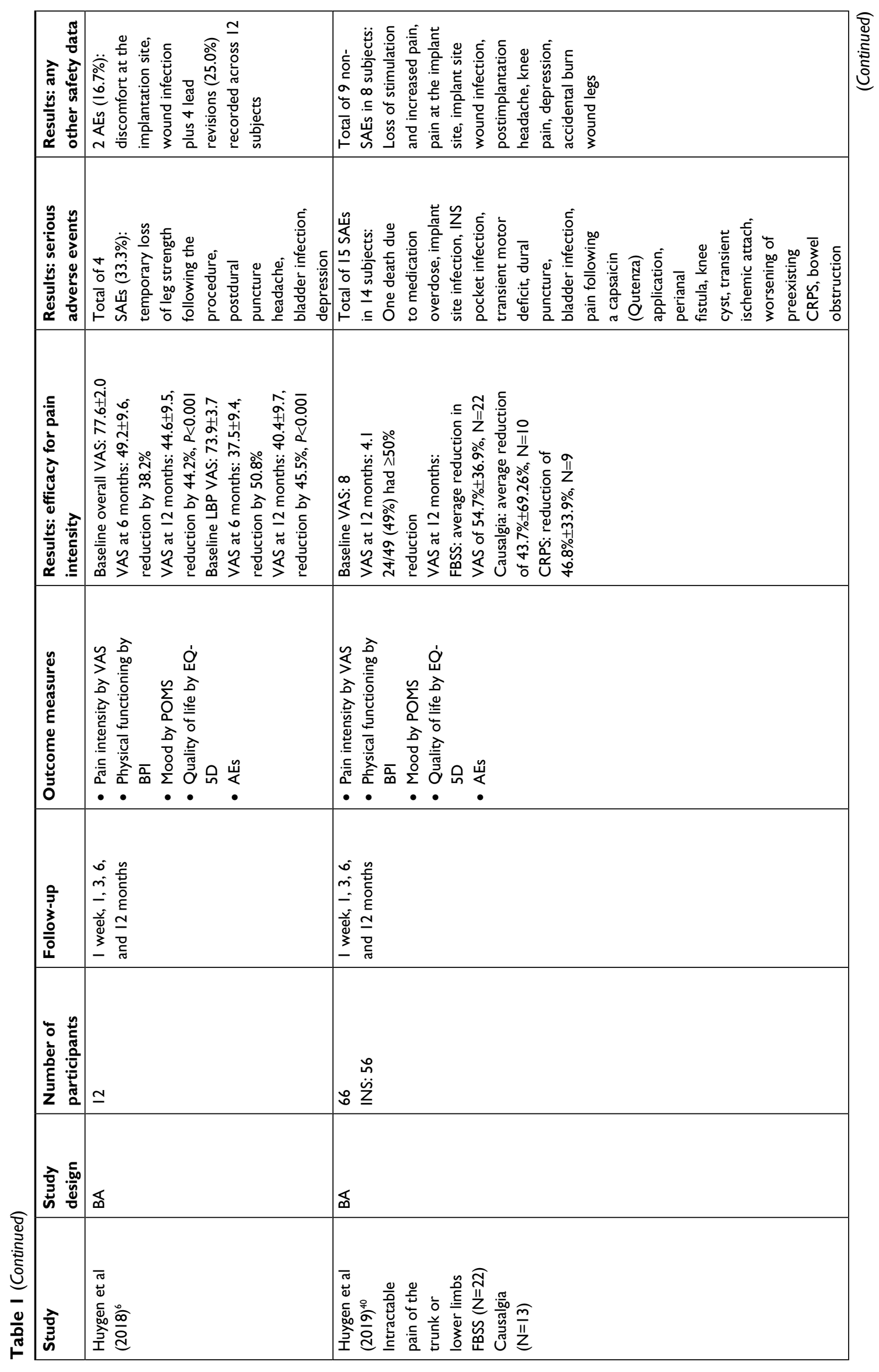




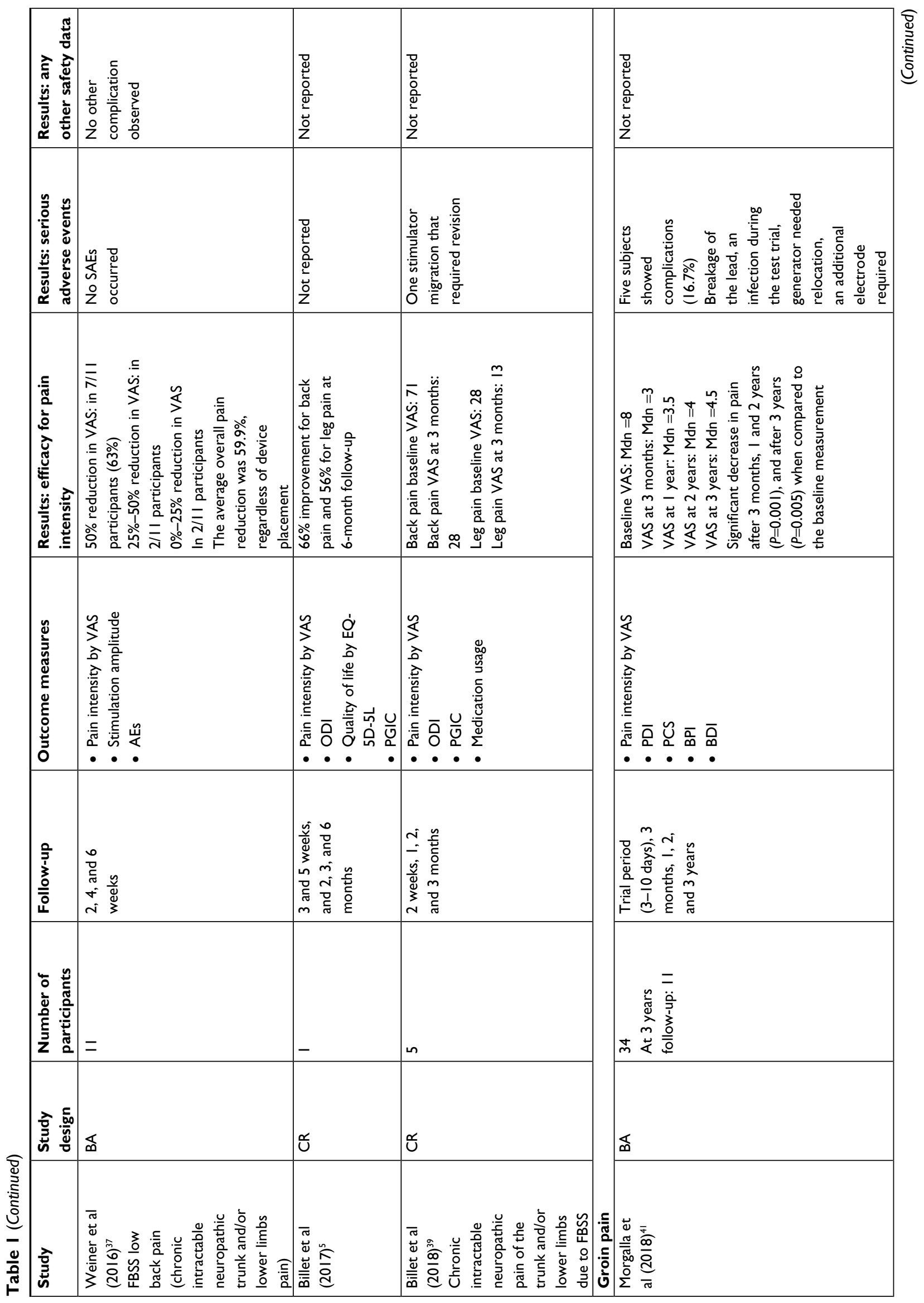




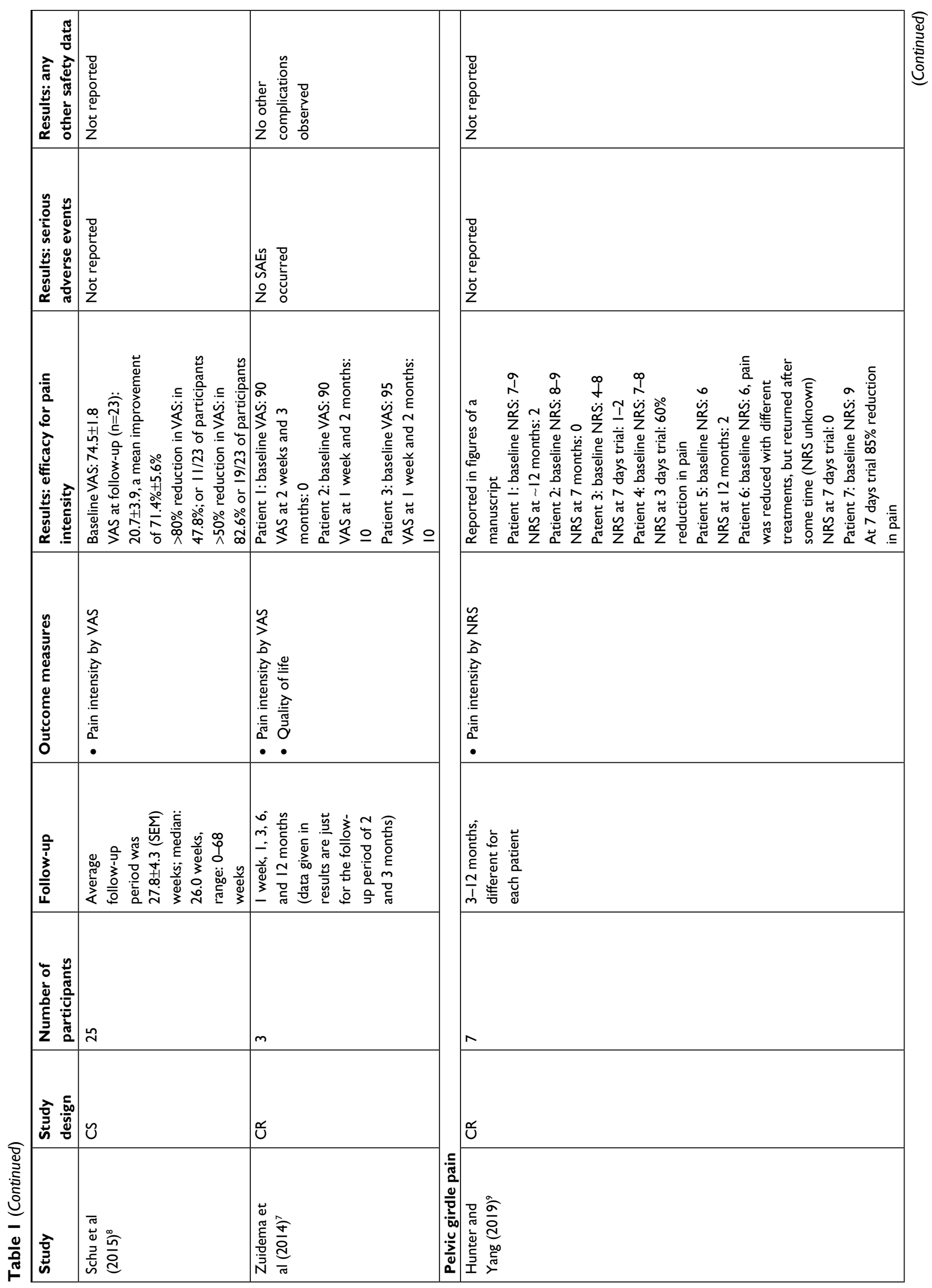




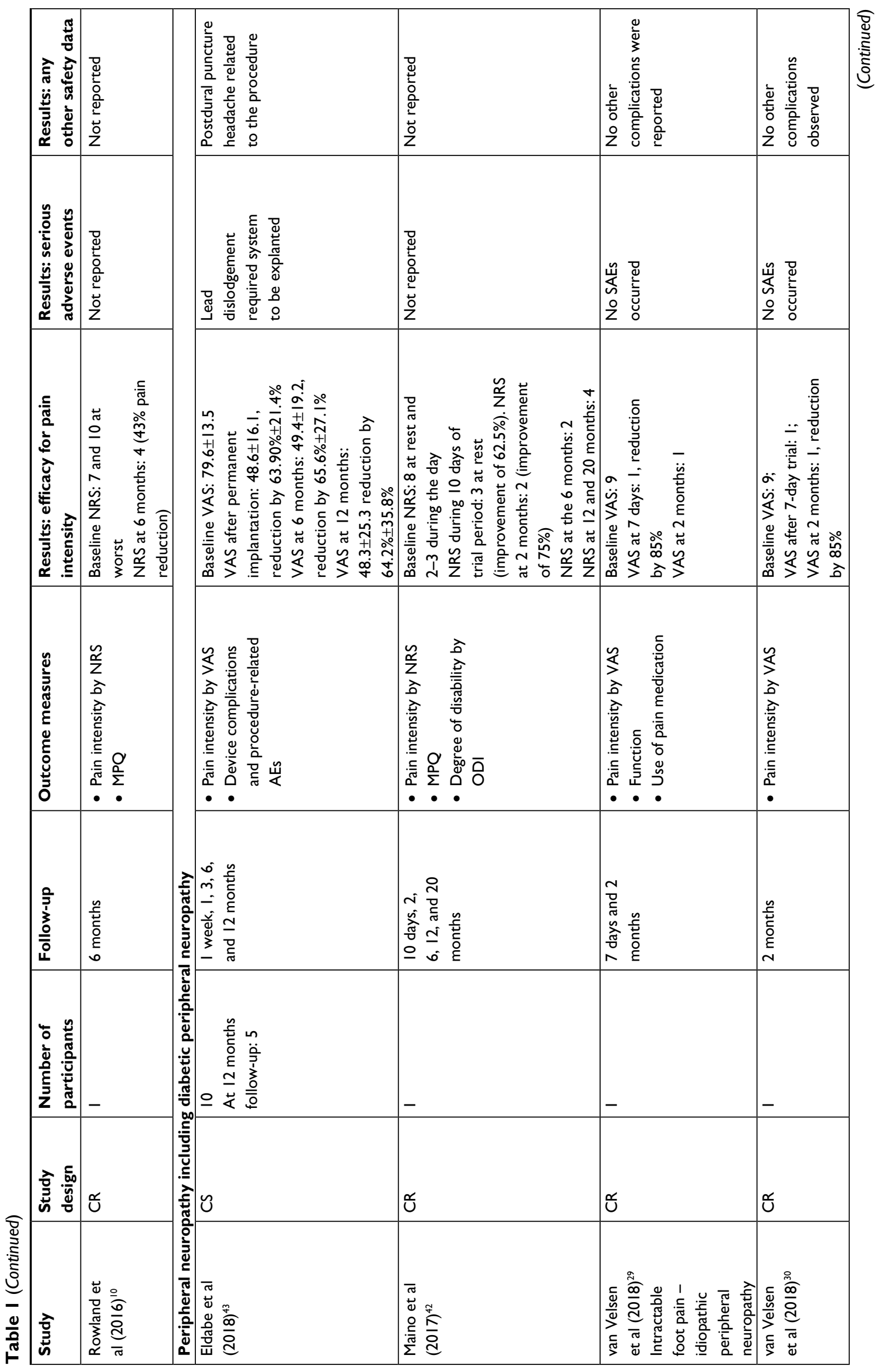




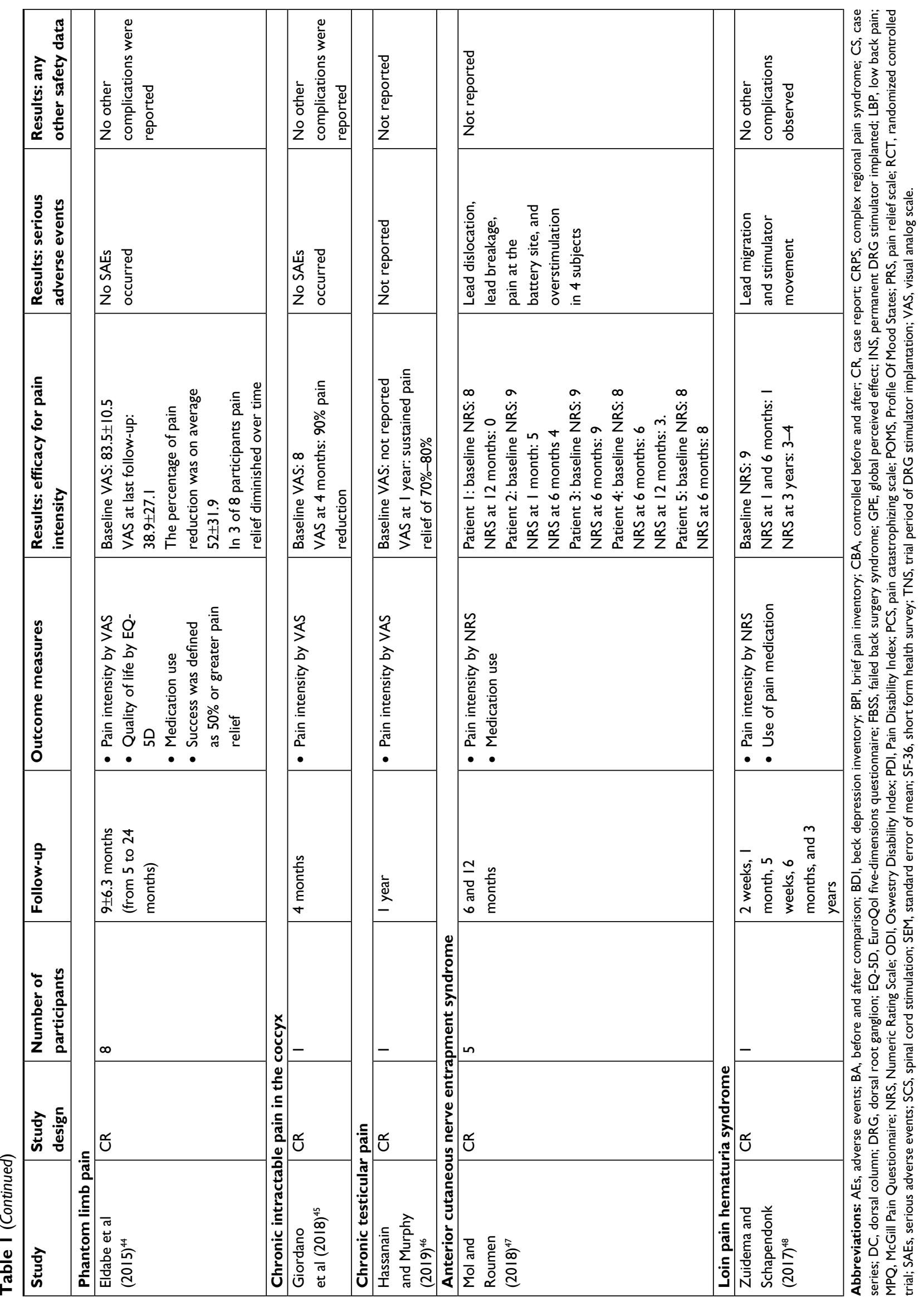


Table 2 Inclusion and exclusion criteria and baseline characteristics of participants

\begin{tabular}{|c|c|c|c|}
\hline Study & $\begin{array}{l}\text { Inclusion criteria/previous } \\
\text { treatment }\end{array}$ & Exclusion criteria & Baseline characteristics \\
\hline \multicolumn{4}{|c|}{ Complex regional pain syndrome } \\
\hline $\begin{array}{l}\text { Deer et al } \\
(2017)^{\prime}\end{array}$ & $\begin{array}{l}\text { - CRPS and/or peripheral } \\
\text { causalgia for at least } 6 \text { months } \\
\text { with chronic, intractable pain } \\
\text { - Age between } 22 \text { and } 75 \text { years } \\
\text { - Naive to stimulation } \\
\text { - Minimum baseline VAS } 60 \mathrm{~mm} \\
\text { in the area of greatest pain } \\
\text { - Failed at least } 2 \text { prior } \\
\text { pharmacologic treatments } \\
\text { from } 2 \text { different drug classes } \\
\text { - Stable neurologic function } 30 \\
\text { days prior to screening } \\
\text { - Free from psychological } \\
\text { pathology that contraindicated } \\
\text { an implantable device }\end{array}$ & $\begin{array}{l}\text { - Back pain was the greatest region of pain } \\
\text { - Pregnant or nursing, plans to become pregnant } \\
\text { - Escalating or changing pain condition } 30 \text { days } \\
\text { prior to study enrollment } \\
\text { - Involved in medically related litigation } \\
\text { - Corticosteroid therapy at an intended site of } \\
\text { stimulation } 30 \text { days or RF } 3 \text { months prior to } \\
\text { study enrollment } \\
\text { - Pain medication(s) dosage(s) was not stable for } \\
\text { - at least } 30 \text { days prior to study enrollment } \\
\text { - Previously failed SCS therapy } \\
\text { - An active implantable device } \\
\text { - Pain only within a cervical distribution } \\
\text { - Cognitive, physical, or sensory impairment } \\
\text { - An indwelling device } \\
\text { - An active systemic infection } \\
\text { - Medical comorbidity that contraindicates } \\
\text { - Placement of device } \\
\text { Participation in another clinical investigation } \\
\text { - } \text { within } 30 \text { days prior to study enrollment } \\
\text { - Diagnosed with cancer within } 2 \text { years prior to } \\
\text { inclusion } \\
\text { - Imaging findings within } 12 \text { months prior to } \\
\text { study enrollment } \\
\text { - Is prisoner }\end{array}$ & $\begin{array}{l}\text { DRG arm: } \\
\text { - } 51.3 \% \text { of females } \\
\text { - } 94.7 \% \text { white race } \\
\text { - Average age } 52.4 \text { years } \\
\text { - Average body mass index } \\
30.5 \mathrm{~kg} / \mathrm{m}^{2} \\
\text { - Average duration of } \\
\text { chronic lower limb pain } \\
7.5 \text { years } \\
\text { SCS arm: } \\
\text { - } 51.3 \% \text { of females } \\
\text { - } 92.1 \% \text { white race } \\
\text { - Average age } 52.5 \text { years } \\
\text { - Average body mass index } \\
28.9 \mathrm{~kg} / \mathrm{m}^{2} \\
\text { - Average duration of } \\
\text { chronic lower limb pain } \\
6.8 \text { years } \\
\text { Comorbidities and } \\
\text { medications taken for subject } \\
\text { conditions were similar in } \\
\text { both arms. No statistically } \\
\text { significant differences were } \\
\text { found among the baseline } \\
\text { characteristics between } \\
\text { treatment arms }\end{array}$ \\
\hline $\begin{array}{l}\text { Liem et al } \\
(20 / 3)^{3}\end{array}$ & $\begin{array}{l}\text { - Chronic, intractable pain in } \\
\text { the trunk, limbs, and/or sacral } \\
\text { region for at least } 6 \text { months } \\
\text { - } \geq 18 \text { years old } \\
\text { - Minimum baseline VAS } 60 \mathrm{~mm} \\
\text { - Failed other treatment } \\
\text { modalities (pharmacological } \\
\text { and/or surgical) } \\
\text { - Have stable pain medication } \\
\text { dosage for a minimum of } 30 \\
\text { days prior to study enrollment } \\
\text { - Have a stable pattern of } \\
\text { neurological symptoms }\end{array}$ & $\begin{array}{l}\text { - Presence of an escalating or changing pain } \\
\text { condition within the month prior to enrollment } \\
\text { - Pain only within a cervical distribution } \\
\text { - Corticosteroid therapy at an intended site of } \\
\text { stimulation within the } 30 \text { days or RF treatment } \\
\text { within the } 3 \text { months prior to study enrollment } \\
\text { - Had a coagulation disorder } \\
\text { - Had an indwelling device } \\
\text { - Had an active implantable device }\end{array}$ & $\begin{array}{l}\text { - } 17 \text { females and } 15 \text { males } \\
\text { - Mean age of men } 58.9 \pm 8.9 \\
\text { years } \\
\text { - Mean age of women } \\
46.9 \pm 12.5 \text { years } \\
\text { - Subjects had chronic pain } \\
\text { of neuropathic origin of } \\
\text { varying etiologies }\end{array}$ \\
\hline $\begin{array}{l}\text { Liem et al } \\
(2015)^{2}\end{array}$ & Same as Liem et al $(2013)^{3}$ & Same as Liem et al $(2013)^{3}$ & Same as Liem et al $(2013)^{3}$ \\
\hline $\begin{array}{l}\text { Goebel et al } \\
(2018)^{34}\end{array}$ & $\begin{array}{l}\text { - Ineffective treatment with } \\
\text { pamidronate, steroids, opioids } \\
\text { - Failed SCS } \\
\text { - Repeated intensive } \\
\text { rehabilitation program with } \\
\text { limited success } \\
\text { - Intravenous immunoglobulin } \\
\text { over a } 6 \text { month period }\end{array}$ & NA & $\begin{array}{l}\text { - Male } \\
\text { (age not written) } \\
\text { - CRPS in a period of } 6 \\
\text { years prior to DRG } \\
\text { stimulation }\end{array}$ \\
\hline $\begin{array}{l}\text { Skaribas et al } \\
(2019)^{36}\end{array}$ & $\begin{array}{l}\text { - Previous back surgery } \\
\text { - Allodynia, hyperpathia, } \\
\text { edema, purplish discoloration } \\
\text { indicating vasomotor changes, } \\
\text { and decreased range of motion } \\
\text { of the affected foot }\end{array}$ & NA & $\begin{array}{l}\text { - } 4 \text { females, I male } \\
\text { - Age between } 49 \text { and } 7 \mathrm{I} \\
\text { years }\end{array}$ \\
\hline
\end{tabular}

(Continued) 
Table 2 (Continued)

\begin{tabular}{|c|c|c|c|}
\hline Study & $\begin{array}{l}\text { Inclusion criteria/previous } \\
\text { treatment }\end{array}$ & Exclusion criteria & Baseline characteristics \\
\hline $\begin{array}{l}\text { van Buyten } \\
\text { et al }(2015)^{35}\end{array}$ & Same as Liem et al $(2013)^{3}$ & Same as Liem et al $(2013)^{3}$ & $\begin{array}{l}\text { - } 6 \text { females, } 2 \text { males } \\
\text { - Average age } 43.9 \pm 5.6 \\
\text { years } \\
\text { - Range: } 18-65 \text { years } \\
\text { - VAS score at baseline } \\
77.9 \pm 4.2 \mathrm{~mm}\end{array}$ \\
\hline $\begin{array}{l}\text { van Bussel et } \\
\text { al }(2018)^{31}\end{array}$ & $\begin{array}{l}\text { - CRPS for a minimum of I year } \\
\text { or longer } \\
\text { - } \geq 18 \text { years old } \\
\text { - Minimum baseline VAS } 50 \mathrm{~mm} \\
\text { or higher } \\
\text { - No improvement in symptoms } \\
\text { after } \geq I \text { year of treatment } \\
\text { according to the Dutch } \\
\text { guidelines for CRPS }\end{array}$ & $\begin{array}{l}\text { - Previous neurostimulation } \\
\text { - Depression or anxiety disorder } \\
\text { - Pregnancy or pregnancy desire within I year } \\
\text { - Body mass index }>35 \mathrm{~kg} / \mathrm{m}^{2} \\
\text { - Life expectancy } \\
\text { - Participants unable to complete the } \\
\text { - questionnaires } \\
\text { - An active implantable device } \\
\text { - Anticoagulant drug therapy or disturbed } \\
\text { - Immunocompromised participants } \\
\text { - Drugs/medication/alcohol addiction }\end{array}$ & $\begin{array}{l}\text { - II females, I male } \\
\text { - Mean age } 38.7 \text { years } \\
\text { (range } 22-57 \text { years) } \\
\text { - VAS score at baseline } 68 \\
\text { mm } \\
\text { - None of the included } \\
\text { subjects had demonstrable } \\
\text { nerve injury in the affected } \\
\text { knee }\end{array}$ \\
\hline $\begin{array}{l}\text { van Bussel et } \\
\text { al }(2015)^{32}\end{array}$ & $\begin{array}{l}\text { - Extensively treated with } \\
\text { different types of oral } \\
\text { medication } \\
\text { - Lumbar sympathetic block } \\
\text { resulted in no clinically } \\
\text { significant relief of symptoms } \\
\text { - Physical therapy failed }\end{array}$ & NA & $\begin{array}{l}\text { - } 48 \text { years old women } \\
\text { - } 5 \text { years of symptoms }\end{array}$ \\
\hline $\begin{array}{l}\text { Yang and } \\
\text { Hunter } \\
(2017)^{33}\end{array}$ & - Failed SCS & NA & $\begin{array}{l}\text { - } 43 \text {-year-old female } \\
\text { - } 2 \text { years of symptoms } \\
\text { - } \text { Baseline NRS 8-9 } \\
\text { - } 50 \text {-year-old female } \\
\text { - } 9 \text { years of symptoms } \\
\text { - } \text { Baseline NRS 8-9 }\end{array}$ \\
\hline \multicolumn{4}{|c|}{ Low back pain } \\
\hline $\begin{array}{l}\text { Deer et al } \\
(2013)^{38}\end{array}$ & $\begin{array}{l}\text { - Chronic, intractable } \\
\text { neuropathic pain of the trunk } \\
\text { and/or limbs } \\
\text { - } \geq 18 \text { years old } \\
\text { - Failed other treatment } \\
\text { modalities including opioid } \\
\text { and nonopioid-based pain } \\
\text { medication, physical therapy, } \\
\text { epidural steroid injections, } \\
\text { selective nerve root blocks, } \\
\text { trigger point injections, } \\
\text { medial branch radiofrequency, } \\
\text { intrathecal pump implantation, } \\
\text { and SCS } \\
\text { - No change in medications, } \\
\text { surgery, injections, or other } \\
\text { treatment for a minimum of } 30 \\
\text { days prior to study enrollment } \\
\text { - Consistent pattern of pain and } \\
\text { neurological symptoms for a } \\
\text { minimum of } 30 \text { days prior to } \\
\text { study enrollment }\end{array}$ & $\begin{array}{l}\text { - Previous posterior fusion } \\
\text { - Severe foraminal stenosis at the expected } \\
\text { target level } \\
\text { - Presence of indwelling implantable devices such } \\
\text { as cardiac devices, spinal cord or peripheral } \\
\text { nerve stimulators, or vascular access catheters } \\
\text { - Pregnancy } \\
\text { Pain patterns could not be }>50 \% \text { in the axial spine, } \\
\text { but axial spine participants were not excluded }\end{array}$ & $\begin{array}{l}\text { - } 5 \text { females, } 5 \text { males } \\
\text { - Average age of men } 52 \pm 5 \\
\text { years } \\
\text { - Average age of women } \\
39 \pm 4 \text { years }\end{array}$ \\
\hline
\end{tabular}

(Continued) 
Table 2 (Continued)

\begin{tabular}{|c|c|c|c|}
\hline Study & $\begin{array}{l}\text { Inclusion criteria/previous } \\
\text { treatment }\end{array}$ & Exclusion criteria & Baseline characteristics \\
\hline $\begin{array}{l}\text { Huygen et al } \\
(2018)^{6}\end{array}$ & $\begin{array}{l}\text { - Back pain due to FBSS } \\
\text { - Axial LBP as either primary or } \\
\text { secondary region of pain } \\
\text { - Minimum baseline VAS } \geq 60 \\
\text { - } \text { Successful DRG stimulation } \\
\text { trial with better than } 50 \% \text { pain } \\
\text { relief } \\
\text { - At least one lead permanently } \\
\text { implanted at an L2 or L3 DRG }\end{array}$ & Not reported & $\begin{array}{l}\text { - } 12 \text { participants, } 33 \% \text { male } \\
\text { - Average age } 5 I . I \text { years } \\
\text { - Baseline VAS } 77.6 \pm 2.0 \\
\text { mm } \\
\text { - All subjects had also leg } \\
\text { pain } \\
\text { - } 6 \text { subjects had foot pain } \\
\text { - } 2 \text { subjects had buttock } \\
\text { pain }\end{array}$ \\
\hline $\begin{array}{l}\text { Huygen et al } \\
(2019)^{40}\end{array}$ & $\begin{array}{l}\text { - } \geq 18 \text { years old } \\
\text { - Chronic pain for at least } \\
6 \text { months } \\
\text { - Minimum baseline VAS } \\
\text { of }>60 \\
\text { - Pain limited to the lower body: } \\
\text { in the thoracic, lumbar, and/or } \\
\text { sacral distributions } \\
\text { - Failed other treatment } \\
\text { modalities including } \\
\text { pharmacological therapy, } \\
\text { physical therapy, and } \\
\text { interventional pain procedures } \\
\text { for chronic pain } \\
\text { - Psychologically appropriate for } \\
\text { the implantation }\end{array}$ & $\begin{array}{l}\text { - Pain primarily in cervical distribution } \\
\text { - Unstable pain condition } \\
\text { - Corticosteroid or radiofrequency treatment at } \\
\text { the intended site of stimulation prior to study } \\
\text { enrollment } \\
\text { - Presence of an active implantable device } \\
\text { - Coagulation disorder or use of anticoagulants } \\
\text { - Cancer } \\
\text { - Pregnancy }\end{array}$ & $\begin{array}{l}\text { - } 64 \% \text { females } \\
\text { - Average age } 52 \pm 11.5 \\
\text { years } \\
\text { - Range from } 30 \text { to } 80 \text { years }\end{array}$ \\
\hline $\begin{array}{l}\text { Weiner et al } \\
(2016)^{37}\end{array}$ & $\begin{array}{l}\text { - Chronic intractable } \\
\text { neuropathic pain of the trunk } \\
\text { and/or lower limbs due to } \\
\text { FBSS } \\
\text { - } \geq 18 \text { years old } \\
\text { - Minimum baseline VAS of }>5 \\
\text { - Speaking English or Spanish } \\
\text { - Pass a psychological evaluation } \\
\text { - Have the cognitive ability } \\
\text { to use the external } \\
\text { transmitter }\end{array}$ & $\begin{array}{l}\text { - Visceral pain } \\
\text { - Hyperalgesia or allodynia of the lower back } \\
\text { - Allergies to system components } \\
\text { - Active cancer treatment } \\
\text { - Drug dependence } \\
\text { - Pregnancy } \\
\text { - Inability to comply with the study requirements }\end{array}$ & $\begin{array}{l}\text { - } 55 \% \text { female } \\
\text { - Mean age } 63 \text { years }\end{array}$ \\
\hline $\begin{array}{l}\text { Billet et al } \\
(2017)^{5}\end{array}$ & $\begin{array}{l}\text { - Microdisectomy with no } \\
\text { results } \\
\text { - Anterior lumbar interbody } \\
\text { fusion performed } \\
\text { - Medical management } \\
\text { with tramadol HCl and } \\
\text { paracetamol } \\
\text { - PRF treatment followed by } \\
\text { ablation of the facet joint did } \\
\text { not provide pain relief }\end{array}$ & NA & $\begin{array}{l}\text { - Patient had traffic accident } \\
\text { resulting in traumatic disc } \\
\text { herniation }\end{array}$ \\
\hline
\end{tabular}

(Continued) 
Table 2 (Continued)

\begin{tabular}{|c|c|c|c|}
\hline Study & $\begin{array}{l}\text { Inclusion criteria/previous } \\
\text { treatment }\end{array}$ & Exclusion criteria & Baseline characteristics \\
\hline $\begin{array}{l}\text { Billet et al } \\
(2018)^{39}\end{array}$ & $\begin{array}{l}\text { - Chronic intractable } \\
\text { neuropathic pain of the trunk } \\
\text { and/or lower limbs due to FBSS } \\
\text { - } \geq 18 \text { years old } \\
\text { - Minimum baseline VAS }>50 \mathrm{~mm} \\
\text { - Refractory to conventional } \\
\text { - } \text { medical management } \\
\text { - Pass a psychological evaluation } \\
\text { - Have the cognitive ability to } \\
\text { - use the external transmitter } \\
\text { - Live within a radius of } 75 \mathrm{~km}\end{array}$ & $\begin{array}{l}\text { - Malignancies, postherpetic neuralgia, active } \\
\text { systemic infection } \\
\text { - Immune-compromised, insulin dependent } \\
\text { - Diabetes not controlled through diet and/or } \\
\text { medication } \\
\text { - Bleeding complications, coagulopathy issues } \\
\text { - Life expectancy of <I year } \\
\text { - Active implanted device } \\
\text { - Pregnancy } \\
\text { - Inability to comply with the study requirements }\end{array}$ & $\begin{array}{l}\text { - } 2 \text { females, } 4 \text { males } \\
\text { - Mean age } 53 \text { years } \\
\text { - Range 33-67 years } \\
\text { - Four subjects reported } \\
\text { also leg pain }\end{array}$ \\
\hline \multicolumn{4}{|l|}{ Groin pain } \\
\hline $\begin{array}{l}\text { Morgalla et } \\
\text { al }(2018)^{41}\end{array}$ & $\begin{array}{l}\text { - Chronic neuropathic pain in } \\
\text { the groin } \\
\text { - Pain confirmed by a clinically } \\
\text { detectable sensory loss, } \\
\text { hyperalgesia, or allodynia, } \\
\text { within an anatomic concordant } \\
\text { area of a nerve or a root } \\
\text { dermatome } \\
\text { - Failure of pain treatment } \\
\text { using various medication, } \\
\text { interventions, or even } \\
\text { hospitalization } \\
\text { - No further indication for } \\
\text { another surgical intervention } \\
\text { in the area of the previously } \\
\text { operated groin }\end{array}$ & $\begin{array}{l}\text { - Previous spinal surgery at the level of the } \\
\text { intended implantation of the DRG leads } \\
\text { - Cardiac pacemakers, vascular access catheters, } \\
\text { other spinal cord stimulators, or peripheral } \\
\text { nerve stimulators (PNSs) } \\
\text { - Psychiatric disorders including anxiety and } \\
\text { depression }\end{array}$ & $\begin{array}{l}\text { - } 13 \text { females, } 21 \text { males } \\
\text { - Mean age } 50.4 \text { years } \\
\text { - Range } 24-84 \text { years } \\
\text { - History of pain for longer } \\
\text { than } 6 \text { months } \\
\text { - Mean duration of pain } 2.5 \\
\text { years, from } 0.5 \text { to } 8 \text { years }\end{array}$ \\
\hline $\begin{array}{l}\text { Schu et al } \\
(2015)^{8}\end{array}$ & $\begin{array}{l}\text { - Chronic, intractable } \\
\text { neuropathic pain of the groin } \\
\text { - } \geq 18 \text { years old } \\
\text { - Failed other treatment } \\
\text { modalities including } \\
\text { oral medications and/or } \\
\text { interventional procedures or } \\
\text { surgical intervention }\end{array}$ & $\begin{array}{l}\text { - Previous posterior fusion } \\
\text { - Severe foraminal stenosis at the expected } \\
\text { target level } \\
\text { - Presence of current indwelling implantable } \\
\text { devices } \\
\text { - Pregnancy }\end{array}$ & $\begin{array}{l}\text { - Mean baseline VAS } \\
(\mathrm{N}=25) 74.5 \pm 1.8 \mathrm{~mm} \\
\text { - The most frequent } \\
\text { diagnosis was } \\
\text { herniorrhaphy }(\mathrm{N}=12) \\
\text { - Other subjects had a } \\
\text { variety of pain etiologies, } \\
\text { many related to } \\
\text { postsurgical pain } \\
\text { - No data about age or sex }\end{array}$ \\
\hline $\begin{array}{l}\text { Zuidema et } \\
\text { al }(20 \mid 4)^{7}\end{array}$ & $\begin{array}{l}\text { - Pain refractory to } \\
\text { antineuropathic medication } \\
\text { (pregabalin and amytriptyline) } \\
\text { - No results with TENS and PRF } \\
\text { and local corticoid infiltration }\end{array}$ & NA & $\begin{array}{l}\text { - } 36 \text { years old men, } 5 \text { years } \\
\text { of chronic pain, baseline } \\
\text { VAS } 90 \mathrm{~mm} \\
\text { - } 39 \text { years old female, } 6 \\
\text { years of chronic pain, } \\
\text { baseline VAS } 90 \mathrm{~mm} \\
\text { - } 46 \text { years old female, } 4 \\
\text { years of chronic pain, } \\
\text { baseline VAS } 95 \mathrm{~mm}\end{array}$ \\
\hline \multicolumn{4}{|c|}{ Pelvic girdle pain } \\
\hline $\begin{array}{l}\text { Hunter and } \\
\text { Yang (2019) }\end{array}$ & $\begin{array}{l}\text { - Pain refractory to medication, } \\
\text { neurolysis, surgery, and, in } \\
\text { some cases, SCS } \\
\text { - Variation in location and } \\
\text { presentation of pain, suspected } \\
\text { cause/etiology, and associated } \\
\text { symptoms }\end{array}$ & NA & $\begin{array}{l}\text { - } 4 \text { females, } 3 \text { males } \\
\text { - Age range from } 36 \text { to } 63 \\
\text { years }\end{array}$ \\
\hline
\end{tabular}

(Continued) 
Table 2 (Continued)

\begin{tabular}{|c|c|c|c|}
\hline Study & $\begin{array}{l}\text { Inclusion criteria/previous } \\
\text { treatment }\end{array}$ & Exclusion criteria & Baseline characteristics \\
\hline $\begin{array}{l}\text { Rowland et } \\
\text { al }(2016)^{10}\end{array}$ & $\begin{array}{l}\text { Failed trials of physiotherapy, } \\
\text { gabapentin, and steroid } \\
\text { injections } \\
\text { On the day of admission, } \\
\text { the patient received oral } \\
\text { treatment with paracetamol } \\
\text { I g four times daily, diazepam } \\
5 \mathrm{mg}, \mathrm{MST} 60 \mathrm{mg} \text { twice } \\
\text { daily, Zomorph (morphine } \\
\text { sulfate) } 10 \mathrm{mg} \text { twice daily and } \\
\text { amitriptyline } 25 \mathrm{mg} \text { once daily }\end{array}$ & NA & $\begin{array}{l}\text { - 37-year-old female } \\
\text { - } 9 \text { years of chronic pain } \\
\text { - Baseline NRS } 7\end{array}$ \\
\hline \multicolumn{4}{|c|}{ Peripheral neuropathy including diabetic peripheral neuropathy } \\
\hline $\begin{array}{l}\text { Eldabe et al } \\
(2018)^{43}\end{array}$ & $\begin{array}{l}\text { - Chronic intractable pain due } \\
\text { to diabetic polyneuropathy of } \\
\text { the lower limbs for at least } 6 \\
\text { months } \\
\text { - } \geq 18 \text { years old } \\
\text { - Stable pain medication for } \\
\text { minimum } 30 \text { days prior to } \\
\text { study enrollment } \\
\text { - Failed previous interventions } \\
\text { including SCS } \\
\text { - Patient primary pain area was } \\
\text { considered any part of the } \\
\text { lower limbs including leg, thigh, } \\
\text { shin, calf, and foot }\end{array}$ & $\begin{array}{l}\text { - Unstable neurological symptoms } \\
\text { - A baseline VAS score of }<60 \mathrm{~mm} \\
\text { - Pregnancy } \\
\text { - Implanted neuromodulation devices }\end{array}$ & $\begin{array}{l}\text { - } 10 \text { male diabetic } \\
\text { participants } \\
\text { - Mean age } 65.2 \text { [SD 8.8] } \\
\text { years } \\
\text { - } \text { mean duration of PDPN- } \\
\text { related symptoms } 7.0(S D \\
\text { 3.I) years, ranging from } 3 \\
\text { to II years ( } \mathrm{n}=5) \\
\text { - Average baseline VAS 79.6 } \\
\text { (SD I3.5) mm }\end{array}$ \\
\hline $\begin{array}{l}\text { Maino et al } \\
(2017)^{42}\end{array}$ & $\begin{array}{l}\text { - No results with multiple } \\
\text { medications including } \\
\text { gabapentin, pregabalin, } \\
\text { duloxetine, amitriptyline, } \\
\text { mirtazapine, lidocaine patches, } \\
\text { topical capsaicin } 8 \% \text {, and } \\
\text { cannabis } \\
\text { - TENS, physical therapy, } \\
\text { acupuncture, and a } \\
\text { corticosteroid infiltration with } \\
\text { no results } \\
\text { - Ice packs and pressure applied } \\
\text { on left foot provided some } \\
\text { relief at night }\end{array}$ & NA & $\begin{array}{l}\text { - 74-year-old men } \\
\text { - Baseline pain NRS } 8 \\
\text { - Chronic pain for } 6 \text { years } \\
\text { - Hyperlipidemia, left } \\
\text { common carotid artery } \\
\text { stenosis, coronary artery } \\
\text { disease, and a depressive } \\
\text { disorder } \\
\text { - Oral daily doses of aspirin } \\
100 \mathrm{mg} \text { and atorvastatin } \\
20 \mathrm{mg}\end{array}$ \\
\hline $\begin{array}{l}\text { van Velsen } \\
\text { et al }(2018)^{30}\end{array}$ & $\begin{array}{l}\text { - Use of neuropathic pain } \\
\text { medications such as } \\
\text { gabapentin, pregabalin, } \\
\text { topiramate, and duloxetine } \\
\text { and opioid analgesics such } \\
\text { ashydrocodone, tapentadol, } \\
\text { oxycodone, and methadone } \\
\text { with no results } \\
\text { - Traditional SCS did not give } \\
\text { satisfactory pain relief }\end{array}$ & NA & $\begin{array}{l}\text { - 45-year-old Caucasian } \\
\text { male patient } \\
\text { - Chronic pain for } 2 \text { years }\end{array}$ \\
\hline $\begin{array}{l}\text { van Velsen } \\
\text { et al }(2018)^{29}\end{array}$ & Same as van Velsen et al $(2018)^{30}$ & NA & $\begin{array}{l}\text { - 45-year-old Caucasian } \\
\text { male patient } \\
\text { - Chronic pain for } 2 \text { years }\end{array}$ \\
\hline
\end{tabular}

(Continued) 
Table 2 (Continued)

\begin{tabular}{|c|c|c|c|}
\hline Study & $\begin{array}{l}\text { Inclusion criteria/previous } \\
\text { treatment }\end{array}$ & Exclusion criteria & Baseline characteristics \\
\hline \multicolumn{4}{|c|}{ Phantom limb pain } \\
\hline $\begin{array}{l}\text { Eldabe et al } \\
(2015)^{44}\end{array}$ & Not reported & Not reported & $\begin{array}{l}\text { - } 5 \text { females and } 3 \text { males } \\
\text { - Age range from } 28 \text { to } 76 \\
\text { years } \\
\text { - Baseline medication } \\
\text { use including clonidine, } \\
\text { bupivacaine, morphine, } \\
\text { pregabalin, oramorph, } \\
\text { amitriptyline, tramadol, } \\
\text { zomorph, lansoprazol, } \\
\text { targin, amineurin, ariclaim, } \\
\text { lyrica, palexia, clonazepam, } \\
\text { oxycodone, gabapentin, } \\
\text { tryptizol, and fentanyl patch }\end{array}$ \\
\hline $\begin{array}{l}\text { Giordano et } \\
\text { al }(2018)^{45}\end{array}$ & $\begin{array}{l}\text { - Multiple coccygeal blocks, } \\
\text { trigger point injections, } \\
\text { epidural steroid injections with } \\
\text { no results } \\
\text { - SCS failed }\end{array}$ & NA & $\begin{array}{l}\text { - } 37 \text {-year-old-female } \\
\text { - } 8 \text { years of chronic pain } \\
\text { - Medication regiment } \\
\text { consisting of oxycodone I0 } \\
\text { mg PO BID, dexketoprofen } \\
25 \mathrm{mg} \text { PO QID, duloxetine } \\
60 \mathrm{mg} \text { PO QD, trazodone } \\
100 \mathrm{mg} \text { PO QD, and } \\
\text { pregabalin } 75 \mathrm{mg} \text { PO BID } \\
\end{array}$ \\
\hline $\begin{array}{l}\text { Hassanain } \\
\text { and Murphy } \\
(2019)^{46}\end{array}$ & $\begin{array}{l}\text { - Trial of antineuropathic medications } \\
\text { in the form of a combination of } \\
\text { amitriptyline and gabapentin } \\
\text { PRF trial for } 3 \text { months (provide } \\
60 \%-70 \% \text { ) pain relief }\end{array}$ & NA & $\begin{array}{l}\text { - } 45 \text {-year-old men } \\
\text { - History of obesity, type } \\
\text { II diabetes mellitus and } \\
\text { obstructive sleep apnea }\end{array}$ \\
\hline $\begin{array}{l}\text { Mol and } \\
\text { Roumen } \\
(2018)^{47}\end{array}$ & $\begin{array}{l}\text { - Patient I: failed neurectomy, } \\
\text { resection of neuroma, use of } \\
\text { opiates } \\
\text { - Patient } 2 \text { : failed neurectomy, } \\
\text { use of pain medications } \\
\text { - Patient } 3 \text { : use of pain } \\
\text { medication } \\
\text { - Patient } 4 \text { : infiltration with } \\
\text { lidocaine, neurectomy } \\
\text { - Patient 5: neurectomy and } \\
\text { TENS }\end{array}$ & NA & $\begin{array}{l}\text { - 35-year-old female, BMI } \\
\text { 35.4, chronic pain for } 6 \\
\text { years, baseline pain NRS } 8 \\
\text { - } 26 \text {-year-old female, BMI } \\
\text { 26.7, chronic pain for } 6 \\
\text { years, baseline pain NRS } 9 \\
\text { - } 50 \text {-year-old men, BMI } 25 . \\
\text { 7, duration of pain not } \\
\text { given, baseline pain NRS } 9 \\
\text { - } 18 \text {-year-old female, BMI } \\
\text { 20. 5, baseline NRS } 8 \\
\text { - 60-year-old men, BMI not } \\
\text { given, chronic pain for } 2 \\
\text { years, baseline NRS } 8 \\
\end{array}$ \\
\hline $\begin{array}{l}\text { Zuidema and } \\
\text { Schapendonk } \\
(2017)^{48}\end{array}$ & $\begin{array}{l}\text { - Use of antineuropathic } \\
\text { analgesics (pregabalin, } \\
\text { amitriptylin, and duloxetin) } \\
\text { - Use of opioid and nonopioid } \\
\text { analgesics (paracetamol, } \\
\text { oxycodone, and tramadol) } \\
\text { - Use of perindopril } \\
\text { - Minimal invasive techniques } \\
\text { (quadratus lumborum block, } \\
\text { repeated neurolytic celiac } \\
\text { plexus blocks, and splanchnic } \\
\text { nerve blocks) with no results } \\
\text { - TENS and catheter-based renal } \\
\text { denervation with no results }\end{array}$ & NA & $\begin{array}{l}\text { - } 37 \text {-year-old women } \\
\text { - Chronic pain for } 2 \text { years } \\
\text { - Patient history: urolithiasis } \\
\text { and tonsillectomy } \\
\text { - Allergy to NSAIDs }\end{array}$ \\
\hline
\end{tabular}

Abbreviations: CRPS, complex regional pain syndrome; DRG, dorsal root ganglion; FBSS, failed back surgery syndrome; LBP, low back pain; NA, not applicable; NRS, Numeric Rating Scale; NSAID, nonsteroidal anti-inflammatory drug; PRF, pulsed radiofrequency; SCS, spinal cord stimulation; TENS, transcutaneous electrical nerve stimulation; VAS, visual analog scale. 
Table 3 Parameters of electrical field stimulation of dorsal root ganglion

\begin{tabular}{|c|c|c|c|c|}
\hline Study & Comparator & $\begin{array}{l}\text { Parameters of } \\
\text { stimulation }\end{array}$ & $\begin{array}{l}\text { Stimulator used (electrode } \\
\text { and device) }\end{array}$ & $\begin{array}{l}\text { Position of the } \\
\text { leads }\end{array}$ \\
\hline \multicolumn{5}{|c|}{ Complex regional pain syndrome } \\
\hline $\begin{array}{l}\text { Deer et al } \\
(2017)^{\prime}\end{array}$ & SCS & 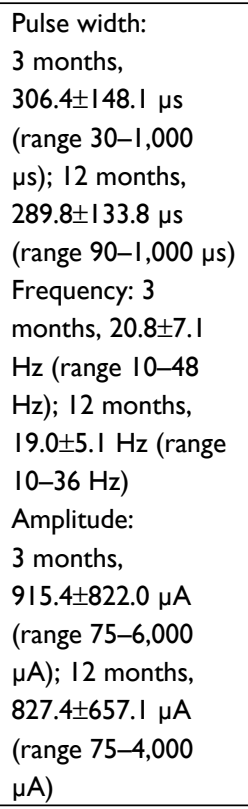 & $\begin{array}{l}\text { For DRG stimulation: Axium } \\
\text { Neurostimulator System (Spinal } \\
\text { Modulation, Inc.) - up to } 4 \text { leads } \\
\text { implanted; for SCS: commercially } \\
\text { available system (Restore Ultra } \\
\text { and Restore Sensor; Medtronic, } \\
\text { Minneapolis, MN, USA) - up to } 2 \\
\text { leads implanted }\end{array}$ & $\begin{array}{l}\text { From TIO to S2 } \\
\text { depending on the } \\
\text { dermatomal target } \\
\text { corresponding } \\
\text { to the subjects' } \\
\text { primary region of } \\
\text { pain }\end{array}$ \\
\hline $\begin{array}{l}\text { Liem et al } \\
(2013)^{3}\end{array}$ & $\begin{array}{l}\text { No } \\
\text { comparator }\end{array}$ & $\begin{array}{l}\text { Pulse width: } 362 \mathrm{~ms} \\
\text { Frequency: } 46 \mathrm{~Hz} \\
\text { Amplitude: } 907 \mu \mathrm{A}\end{array}$ & $\begin{array}{l}\text { Axium neurostimulator and } 4 \\
\text { quadrupolar percutaneous leads } \\
\text { and wireless programmer devices } \\
\text { (Spinal Modulation, Inc.) }\end{array}$ & $\begin{array}{l}\text { According to } \\
\text { individual location } \\
\text { and distribution of } \\
\text { pain (not specified) }\end{array}$ \\
\hline $\begin{array}{l}\text { Liem et al } \\
(2015)^{2}\end{array}$ & $\begin{array}{l}\text { No } \\
\text { comparator }\end{array}$ & $\begin{array}{l}\text { Pulse width: } 362 \mathrm{~ms} \\
\text { Frequency: } 46 \mathrm{~Hz} \\
\text { Amplitude: } 907 \mu \mathrm{A}\end{array}$ & $\begin{array}{l}\text { Axium neurostimulator and } 4 \\
\text { quadrupolar percutaneous leads } \\
\text { and wireless programmer devices } \\
\text { (Spinal Modulation, Inc.) }\end{array}$ & $\begin{array}{l}\text { According to } \\
\text { individual location } \\
\text { and distribution of } \\
\text { pain (not specified) }\end{array}$ \\
\hline $\begin{array}{l}\text { van Bussel } \\
\text { et al }(2018)^{31}\end{array}$ & SCS & $\begin{array}{l}\text { Not written in } \\
\text { manuscript }\end{array}$ & $\begin{array}{l}\text { Two 4-contact leads and DRG } \\
\text { stimulator (St. Jude Medical Inc., } \\
\text { Little Canada, MN, USA) }\end{array}$ & L3 and L4 DRG \\
\hline $\begin{array}{l}\text { Goebel et al } \\
(2018)^{34}\end{array}$ & $\begin{array}{l}\text { No } \\
\text { comparator }\end{array}$ & $\begin{array}{l}\text { Not written in } \\
\text { manuscript }\end{array}$ & Not written in manuscript & L4 DRG \\
\hline $\begin{array}{l}\text { Skaribas et al } \\
(2019)^{36}\end{array}$ & $\begin{array}{l}\text { No } \\
\text { comparator }\end{array}$ & $\begin{array}{l}\text { Not written in } \\
\text { manuscript }\end{array}$ & Two quadrupolar DRG electrodes & SI \\
\hline $\begin{array}{l}\text { van Bussel } \\
\text { et al }(2015)^{32}\end{array}$ & $\begin{array}{l}\text { No } \\
\text { comparator }\end{array}$ & $\begin{array}{l}\text { Pulse width: I } 70 \mu \mathrm{s} \\
\text { for lead I and } 2 \text { (L2 } \\
\text { and L3) and I } 60 \mu \mathrm{s} \\
\text { for lead } 3(\mathrm{~L} 42) \\
\text { Frequency: } 20 \mathrm{~Hz} \\
\text { Amplitude: } L 2,700 \\
\mu \mathrm{A} ; \mathrm{L} 3, \mathrm{I}, 030 \mu \mathrm{A} \text {; } \\
\mathrm{L} 4,500 \mu \mathrm{A}\end{array}$ & $\begin{array}{l}\text { Three quadrupolar DRG } \\
\text { stimulation leads (refer to Liem et } \\
\text { al (20I3); without giving company } \\
\text { or stimulator details) }\end{array}$ & L2-L4 \\
\hline $\begin{array}{l}\text { van Buyten } \\
\text { et al }(2015)^{35}\end{array}$ & $\begin{array}{l}\text { No } \\
\text { comparator }\end{array}$ & $\begin{array}{l}\text { Not written in } \\
\text { manuscript }\end{array}$ & $\begin{array}{l}\text { Quadrupolar percutaneous leads } \\
\text { and Axium stimulator (Spinal } \\
\text { Modulation, Inc.) }\end{array}$ & $\begin{array}{l}\text { According to } \\
\text { individual location } \\
\text { and distribution } \\
\text { of pain }\end{array}$ \\
\hline $\begin{array}{l}\text { Yang and } \\
\text { Hunter } \\
(2017)^{33}\end{array}$ & SCS & $\begin{array}{l}\text { Not written in } \\
\text { manuscript }\end{array}$ & $\begin{array}{l}\text { Axium stimulator (Spinal } \\
\text { Modulation, Inc.) }\end{array}$ & $\mathrm{L} 3$ and $\mathrm{L} 4$ \\
\hline
\end{tabular}

(Continued) 
Table 3 (Continued)

\begin{tabular}{|c|c|c|c|c|}
\hline Study & Comparator & $\begin{array}{l}\text { Parameters of } \\
\text { stimulation }\end{array}$ & $\begin{array}{l}\text { Stimulator used (electrode } \\
\text { and device) }\end{array}$ & $\begin{array}{l}\text { Position of the } \\
\text { leads }\end{array}$ \\
\hline \multicolumn{5}{|c|}{ Low back pain } \\
\hline $\begin{array}{l}\text { Deer et al } \\
(2013)^{38}\end{array}$ & $\begin{array}{l}\text { No } \\
\text { comparator }\end{array}$ & $\begin{array}{l}\text { Pulse width: } 200 \mu \mathrm{s} ; \\
\text { Frequency: } 68 \mathrm{~Hz} \text {; } \\
\text { Amplitude: } 800 \mu \mathrm{A}\end{array}$ & $\begin{array}{l}\text { Quadrupolar DRG stimulation } \\
\text { leads (Spinal Modulation, Inc.) } \\
\text { and external stimulator (Spinal } \\
\text { Modulation, Inc) }\end{array}$ & $\begin{array}{l}\text { Thoracic, lumbar, } \\
\text { and sacral spinal } \\
\text { levels }\end{array}$ \\
\hline $\begin{array}{l}\text { Huygen et al } \\
(2018)^{6}\end{array}$ & $\begin{array}{l}\text { No } \\
\text { comparator }\end{array}$ & $\begin{array}{l}\text { Pulse width: } \\
269 \pm 17.0 \mu \mathrm{s}, \text { range } \\
80-440 \mu \mathrm{s} \\
\text { Frequency: } 21.3 \pm 0.6 \\
\mathrm{~Hz} \text {, range } 20-30 \mathrm{~Hz} \\
\text { Amplitude: } \\
591.9 \pm 50.3 \mu \mathrm{A} \text {, range } \\
\mathrm{I}, 750-\mathrm{I}, 130 \mu \mathrm{A}\end{array}$ & $\begin{array}{l}\text { Axium neurostimulator (Spinal } \\
\text { Modulation, Inc.) - up to } 4 \text { leads } \\
\text { were implanted per subject, leads } \\
\text { in bipolar configuration }\end{array}$ & $\begin{array}{l}\text { L2 or L3 DRG. } \\
\text { One lead was } \\
\text { placed at each of } \\
\text { the LI, L4, L5, } \\
\text { and SI DRGs in } \\
\text { subjects with foot } \\
\text { and buttock pain }\end{array}$ \\
\hline $\begin{array}{l}\text { Huygen et al } \\
(2019)^{40}\end{array}$ & $\begin{array}{l}\text { No } \\
\text { comparator }\end{array}$ & $\begin{array}{l}\text { Not written in } \\
\text { manuscript }\end{array}$ & $\begin{array}{l}\text { Axium neurostimulator (Abbott } \\
\text { Laboratories) }\end{array}$ & $\begin{array}{l}\text { Not written in } \\
\text { manuscript }\end{array}$ \\
\hline $\begin{array}{l}\text { Weiner et al } \\
(2016)^{37}\end{array}$ & $\begin{array}{l}\text { No } \\
\text { comparator }\end{array}$ & $\begin{array}{l}\text { Pulse width: } 500 \mu \mathrm{s} \\
\text { Frequency: } 100 \mathrm{~Hz}\end{array}$ & $\begin{array}{l}\text { The Stimwave Freedom SCS } \\
\text { System, including stimulator and } \\
\text { electrode (Stimwave Technologies } \\
\text { Incorporated, Fort Lauderdale, } \\
\text { FL, USA) }\end{array}$ & LI-L5 \\
\hline $\begin{array}{l}\text { Billet et al } \\
(2017)^{5}\end{array}$ & $\begin{array}{l}\text { No } \\
\text { comparator }\end{array}$ & $\begin{array}{l}\text { Pulse width: } 30 \mu \mathrm{s} \\
\text { Frequency: } 10 \mathrm{kHz} \\
\text { Amplitude: } 1.5 \text { and } \\
2.5 \mathrm{~mA}\end{array}$ & $\begin{array}{l}\text { Two Freedom 4A electrodes with } \\
\text { four contacts and Freedom SCS } \\
\text { external device (Stim Relieve LLC) }\end{array}$ & $\mathrm{L} 2$ \\
\hline $\begin{array}{l}\text { Billet et al } \\
(2018)^{39}\end{array}$ & $\begin{array}{l}\text { No } \\
\text { comparator }\end{array}$ & 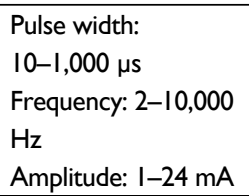 & $\begin{array}{l}\text { Two Freedom 4A electrodes with } \\
\text { four contacts; each electrode } \\
\text { array contains four contacts } \\
\text { ( } 3 \mathrm{~mm} \text { in diameter with } 4 \mathrm{~mm} \\
\text { spacing) (Stimwave) }\end{array}$ & T9 and $\mathrm{L} 2$ \\
\hline \multicolumn{5}{|l|}{ Groin pain } \\
\hline $\begin{array}{l}\text { Morgalla } \\
\text { et al }(2018)^{41}\end{array}$ & $\begin{array}{l}\text { No } \\
\text { comparator }\end{array}$ & $\begin{array}{l}\text { Not written in } \\
\text { manuscript }\end{array}$ & Not written in manuscript & $\begin{array}{l}\text { TI2, LI, and } \\
\text { L2 (mostly } \\
\text { combination of LI } \\
\text { and L2) }\end{array}$ \\
\hline $\begin{array}{l}\text { Schu et al } \\
(2015)^{8}\end{array}$ & $\begin{array}{l}\text { No } \\
\text { comparator }\end{array}$ & $\begin{array}{l}\text { Pulse width: } 137 \mathrm{~ms} \\
\text { Frequency: } 60 \mathrm{~Hz} \\
\text { Amplitude: } 6.32 \mathrm{~mA}\end{array}$ & $\begin{array}{l}\text { Axium neurostimulator system } \\
\text { and quadrupolar DRG stimulation } \\
\text { leads (Spinal Modulation, Inc.) }\end{array}$ & $\begin{array}{l}\text { TII up to L3; } \\
\text { Subjects received } \\
\text { I, } 2 \text {, or } 3 \text { leads to } \\
\text { cover their pain } \\
\text { area. All leads were } \\
\text { placed unilaterally }\end{array}$ \\
\hline $\begin{array}{l}\text { Zuidema } \\
\text { et al }(20 \mid 4)^{7}\end{array}$ & $\begin{array}{l}\text { No } \\
\text { comparator }\end{array}$ & $\begin{array}{l}\text { Not written in } \\
\text { manuscript }\end{array}$ & $\begin{array}{l}\text { Axium stimulator and DRG lead } \\
\text { (Spinal Modulation, Inc.) }\end{array}$ & $\mathrm{TI}$, TI2, and L2 \\
\hline \multicolumn{5}{|c|}{ Pelvic girdle pain } \\
\hline $\begin{array}{l}\text { Hunter and } \\
\text { Yang }(2019)^{9}\end{array}$ & $\begin{array}{l}\text { No } \\
\text { comparator }\end{array}$ & $\begin{array}{l}\text { Not written in } \\
\text { manuscript }\end{array}$ & Not written in manuscript & $\mathrm{LI}$ and $\mathrm{S} 2$ \\
\hline $\begin{array}{l}\text { Rowland } \\
\text { et al }(2016)^{10}\end{array}$ & $\begin{array}{l}\text { No } \\
\text { comparator }\end{array}$ & $\begin{array}{l}\text { LI: } \\
\text { Pulse width: } \\
200-530 \mu \mathrm{s} \\
\text { Frequency: } 20-40 \\
\mathrm{~Hz} \\
\text { Amplitude: } 575-650 \\
\mu \mathrm{A} \\
\text { L2: } \\
\text { Pulse width: } 300 \mathrm{~ms} \\
\text { Frequency: } 20-40 \\
\text { Hz } \\
\text { Amplitude: } 750 \mathrm{mV}\end{array}$ & $\begin{array}{l}2 \text { leads were implanted; no } \\
\text { details given about company or } \\
\text { stimulator }\end{array}$ & $\mathrm{LI}$ and $\mathrm{L} 2$ \\
\hline
\end{tabular}


Table 3 (Continued)

\begin{tabular}{|c|c|c|c|c|}
\hline Study & Comparator & $\begin{array}{l}\text { Parameters of } \\
\text { stimulation }\end{array}$ & $\begin{array}{l}\text { Stimulator used (electrode } \\
\text { and device) }\end{array}$ & $\begin{array}{l}\text { Position of the } \\
\text { leads }\end{array}$ \\
\hline \multicolumn{5}{|c|}{ Peripheral neuropathy including diabetic peripheral neuropathy } \\
\hline $\begin{array}{l}\text { Eldabe et al } \\
(2018)^{43}\end{array}$ & $\begin{array}{l}\text { No } \\
\text { comparator }\end{array}$ & $\begin{array}{l}\text { Based on patients' } \\
\text { feedback, stimulation } \\
\text { was programmed for } \\
\text { either subperception } \\
\text { or paraesthesia with } \\
\text { participants able to } \\
\text { adjust parameters } \\
\text { using a wireless } \\
\text { controller }\end{array}$ & $\begin{array}{l}\text { Up to } 4 \text { quadrupolar leads and } \\
\text { fully implantable neurostimulation } \\
\text { system (Abbott Laboratories) }\end{array}$ & $\mathrm{L} 2$ and $\mathrm{L} 5$ \\
\hline $\begin{array}{l}\text { Maino et al } \\
(2017)^{42}\end{array}$ & $\begin{array}{l}\text { No } \\
\text { comparator }\end{array}$ & $\begin{array}{l}\text { Pulse width: } 670 \mu \mathrm{s} \\
\text { Frequency: } 40 \mathrm{~Hz} \\
\text { Amplitude: } 1.88 \mathrm{~mA}\end{array}$ & $\begin{array}{l}\text { Quadrupolar DRG lead (Axium, } \\
\text { Spinal Modulation, Inc) }\end{array}$ & L5 \\
\hline $\begin{array}{l}\text { van Velsen } \\
\text { et al }(2018)^{29}\end{array}$ & $\begin{array}{l}\text { No } \\
\text { comparator }\end{array}$ & $\begin{array}{l}\text { Not written in } \\
\text { manuscript }\end{array}$ & $\begin{array}{l}\text { 4-contact Axium }{ }^{\mathrm{TM}} \text { lead (St. Jude } \\
\text { Medical, Plano, TX, USA) }\end{array}$ & L5 and SI \\
\hline $\begin{array}{l}\text { van Velsen } \\
\text { et al }(2018)^{30}\end{array}$ & $\begin{array}{l}\text { No } \\
\text { comparator }\end{array}$ & $\begin{array}{l}\text { Not written in } \\
\text { manuscript }\end{array}$ & $\begin{array}{l}\text { Bilateral leads, } 4 \text { leads in total } \\
\text { (4-contact Axium lead, St Jude } \\
\text { Medical, St Paul, MN, USA), } \\
\text { stimulator not specified }\end{array}$ & L5 and SI \\
\hline \multicolumn{5}{|c|}{ Chronic testicular pain } \\
\hline $\begin{array}{l}\text { Hassanain } \\
\text { and Murphy } \\
(20 / 8)^{46}\end{array}$ & $\begin{array}{l}\text { No } \\
\text { comparator }\end{array}$ & $\begin{array}{l}\text { Pulse width: } 130 \mu \mathrm{s} \\
\text { Frequency: } 20 \mathrm{~Hz} \\
\text { Amplitude: } 0.55-0.6 \\
\mathrm{~mA}\end{array}$ & $\begin{array}{l}\text { Proclaim DRG } \\
\text { Implantable Pulse Generator IPG } \\
\text { (Abbott Laboratories) and Axium } \\
\text { Neurostimulator System Slim Tip } \\
\text { A50 leads (Abbott Laboratories) }\end{array}$ & LI \\
\hline \multicolumn{5}{|c|}{ Anterior cutaneous nerve entrapment syndrome } \\
\hline $\begin{array}{l}\text { Mol and } \\
\text { Roumen } \\
(2018)^{47}\end{array}$ & $\begin{array}{l}\text { No } \\
\text { comparator }\end{array}$ & $\begin{array}{l}\text { Not written in } \\
\text { manuscript }\end{array}$ & DRG Axium Neurostimulator & T9-TI2 and L2 \\
\hline \multicolumn{5}{|c|}{ Loin pain hematuria syndrome } \\
\hline $\begin{array}{l}\text { Zuidema and } \\
\text { Schapendonk } \\
(2017)^{48}\end{array}$ & $\begin{array}{l}\text { No } \\
\text { comparator }\end{array}$ & $\begin{array}{l}\text { TI 2: } \\
\text { Pulse width: } 300 \text {, } \\
\text { I30, I } 80 \mathrm{~ms} \\
\text { Frequency: } 20 \text { and } \\
24 \mathrm{~Hz} \\
\text { Amplitude: } 0.7,0.95 \text {, } \\
\text { and } 0.775 \mathrm{~mA} \\
\text { LI: } \\
\text { Pulse width: } 300 \text {, } \\
\text { I30, I40, and } 180 \mathrm{~ms} \\
\text { Frequency: } 20 \text { and } \\
24 \mathrm{~Hz} \\
\text { Amplitude: } 0.18,0.6 \text {, } \\
0.7 \text {, and } 0.2 \mathrm{~mA}\end{array}$ & $\begin{array}{l}\text { Axium permanent stimulator } \\
\text { and quadrupolar stimulation lead } \\
\text { (Spinal Modulation, Inc) }\end{array}$ & $\mathrm{TI} 2$ and $\mathrm{LI}$ \\
\hline
\end{tabular}

Abbreviations: CRPS, complex regional pain syndrome; DRG, dorsal root ganglion.

fied as active or recruiting with no results (details are given in Table S3).

\section{Results for efficacy and selection of participants for different pain syndromes Complex regional pain syndrome}

CRPS was the most common indication treated with EFS neuromodulation of DRG among studies included in this review, with nine such studies (Table 1). ACCURATE, an RCT published in 2017, included 152 participants and compared neuromodulation with EFS of DRG with traditional SCS. ${ }^{1}$ The remaining studies had nonrandomized designs. Three studies were before and after comparisons, including a total of 71 participants during the trial period of stimulation and 44 for permanent implantation. ${ }^{2,3,31}$ There were five case reports that included 17 participants in total. ${ }^{32-36}$ 
The ACCURATE trial by Deer et al included participants suffering from chronic, intractable pain for at least 6 months, who have tried and failed at least two prior pharmacologic treatments from two different drug classes. ${ }^{1}$ The trial results showed that the proportion of participants who achieved treatment success at 3 months in DRG EFS neuromodulation group of participants (81\%) was statistically higher compared to the group treated with SCS (56\%). A similar result was observed at 12 months follow-up when $74 \%$ of participants in DRG EFS neuromodulation group and 53\% in SCS group still had significant pain relief. ${ }^{1}$ For both the follow-up time points, the results demonstrated DRG stimulation statistical noninferiority $(P<0.0001)$ but also statistical superiority $(P<0.0004)$. DRG stimulation also demonstrated greater improvements in quality of life and psychological disposition when compared to SCS. ${ }^{1}$ Beside CRPS, 32 participants in DRG arm and 33 in SCS arm were diagnosed with causalgia. At 3 months follow-up, when results were stratified by primary diagnosis, higher proportion of participants from DRG arm (79.3\%) met the primary endpoint, in comparison with SCS arm (53.3\%). ${ }^{1}$

Two uncontrolled before-after comparisons from Liem et al included participants with chronic intractable pain who have failed other treatment modalities (pharmacological and/ or surgical) and followed them for 6 months $^{3}$ and 12 months ${ }^{2}$ after a permanent stimulator implantation. Results showed pain reduction by $66.1 \%$ from baseline immediately after implantation of permanent stimulator and remained stable at $6(P<0.001)$ and 12 months $(P<0.005)$ follow-up visit, with $56.1 \%$ reduction from baseline. These studies also measured the psychological aspects of pain management using 30-item Brief Profile of Mood States (POMS) and showed statistically significant improvement in four out of six domains of the POMS, as well as decrease in the total mood disturbance score. ${ }^{2,3}$ Third before-after study by van Bussel et al in 2018 compared the efficacy of dorsal column (DC) stimulation vs DRG EFS neuromodulation. Participants had a trial period of 16 days with two stimulation types, SCS and DRG. Reduction of pain was comparable between two groups, but most of the participants preferred DRG stimulation $(P=0.04)$ since they did not feel stimulation-induced paresthesia and did not have to adjust stimulation intensity during the day, which was necessary for SCS stimulation. ${ }^{31}$

Among the five case reports, Yang et al (2017) reported 2 cases of implantation of DRG stimulation system after the failure of traditional SCS, in which both the participants reported sustained pain relief at 8 months follow-up. ${ }^{33} \mathrm{~A}$ case report of van Bussel et al (2015) included one participant, followed for 3 months; major pain relief was reported after 8 days, 1 and 3 months. ${ }^{32}$ The remaining three case reports included a total of 17 participants who have previously failed various treatment; they measured pain intensity by numeric rating scale (NRS); and all participants showed $>50 \%$ pain reduction at all follow-up time points (Table 1). ${ }^{34-36}$

Analysis of participants with CRPS included in studies warrants division of those studies in two groups, where one group included participants in an RCT or an observational study with clear inclusion and exclusion criteria and another group included participants reported in a case series or case report without such criteria.

In studies with defined inclusion and exclusion criteria, common criteria for inclusion were that participants were aged above 18 years, diagnosed with CRPS for at least 6 months, had visual analog scale (VAS) scores of at least 50 or $60 \mathrm{~mm}$ out of $100 \mathrm{~mm}$, had failed previous treatment including pharmacological and surgical, were naive to stimulation, had stable neurologic function, and were free from psychological pathology that contraindicated an implantable device (Table 2). Exclusion criteria were participants who already had an implantable device, had previously failed SCS therapy, had cognitive, physical, or sensory impairment, had a coagulation disorder or uses anticoagulants, and if pregnant or planning pregnancy (Table 2). On the contrary, in all case studies and case reports, DRG stimulation was the last treatment option when participants failed all other treatment modalities including also, in some cases, SCS (Table 2). Generally, participants included in these nine studies were older than 50 years, and only one study reported race of participants, with $>90 \%$ of white participants included. Therefore, the results of these studies are not necessarily generalizable of the population suffering from CRPS.

We also found considerable heterogeneity in terms of stimulation parameters for neuromodulation with EFS that were used in these studies. Stimulation leads were implanted mostly according to dermatomal target corresponding to the participants' primary region of pain and included levels from T10 to S2, but mostly at the levels L3 and L4 (Table 3). Stimulation parameters varied among the studies. Programming of stimulator in ACCURATE trial was performed by experienced personnel to achieve optimal analgesia so parameters changed at different time points. Average pulse width at 3 months was $306.4 \pm 148.1 \mu \mathrm{s}$, while at 12 months it was $289.8 \pm 133.8 \mu \mathrm{s}$. Average frequency was similar, $20.8 \pm 7.1 \mathrm{~Hz}$ and $19.0 \pm 5.1 \mathrm{~Hz}$ for 3 and 12 months, respectively. Amplitude had the widest range with average values of $915.4 \pm 822.0$ $\mu \mathrm{A}$ for 3 months and $827.4 \pm 657.1 \mu \mathrm{A}$ for 12 months. ${ }^{1}$ Other 
studies had fixed parameters with pulse width between $160 \mu \mathrm{s}$ and $362 \mathrm{~ms}$, frequency between 20 and $46 \mathrm{~Hz}$, and amplitude between 500 and $1,030 \mu \mathrm{A}$. Details of neuromodulation with EFS parameters used in analyzed studies are given in Table 3.

\section{Low back pain}

Four uncontrolled before-after studies included 33 participants with LBP. ${ }^{6,37,38,40}$ Deer et al (2013) included 10 participants with pain of different etiologies (including peripheral neuropathy and postherpetic neuralgia), but most of the participants had LBP, so we included it in this group (Table 2).$^{38}$ The average reduction in pain between baseline and final visit was $70 \% \pm 32 \%(P=0.0007)$. Time of last follow-up for different participants varied from 6 months up to 2 years. ${ }^{38}$

Uncontrolled before-after studies of Huygen et al and Weiner et al included participants with LBP after failed back surgery syndrome (FBSS). Huygen et al (2018) found that at 12 months follow-up, VAS was reduced by $44.2 \%(P<0.001)$ from baseline. ${ }^{6}$ The other study of Huygen et al included participants with multiple etiologies; 25 participants with LPB due the FBSS, 13 diagnosed with causalgia, 11 with CRPS, and few participants with several others etiologies. From 56 included participants, 49 who were implanted with permanent stimulation system were followed up to 12 months, $49 \%$ of them reported $\geq 50 \%$ pain relief, whereas $82 \%$ reported at least $30 \%$ reduction from baseline $(P<0.0001) .{ }^{40}$ Both the studies reported sustained improvements in mood measured by POMS. ${ }^{6,40}$ In Weiner et al (2016), after 6 weeks $>50 \%$ reduction in VAS was achieved in $63 \%$ of participants. ${ }^{37}$ Parameters of stimulation and details about stimulator used are given in Table 3.

Two studies in this group were case reports that used a novel high-frequency type of neuromodulation with EFS with parameters of stimulation different from standard DRG stimulation. One was case report including only one patient with fixed stimulation parameters that showed $66 \%$ improvement for back pain and 56\% for leg pain at 6 months followup. ${ }^{5}$ The other was a small feasibility study that included six participants and used a range of stimulation parameters, specifically amplitude between 1 and $24 \mathrm{~mA}$, pulse width of $10-1000 \mu \mathrm{s}$, and frequency of $2-10,000 \mathrm{~Hz}$. The study reported that all participants achieved $>50 \%$ pain reduction from baseline to 3 months follow-up (Table 3). ${ }^{39}$

Main inclusion criteria for participants in this group of studies were at least 18 years old, diagnosed chronic pain syndrome, VAS scores $>5$ or 6 out of 10 , failure of other treatment modalities. Participants were excluded if they had an active implantable stimulator of any type, if pregnant, and if they had any inability to comply with study requirements (Table 2).

\section{Groin pain}

Groin pain was analyzed in one uncontrolled before-after comparison with 34 participants, ${ }^{41}$ one case series with 25 participants suffering from groin pain of different etiology and one case report describing three participants. ${ }^{7}$ Morgalla et al had follow-up for as much as 3 years and showed significant decrease in pain after 3 months, after 1 and 2 years $(P=0.001)$, and after 3 years $(P=0.005)$, when compared to the baseline measurement. ${ }^{41} \mathrm{Sch} u$ et al reported $>80 \%$ reduction in VAS in $47.8 \%$ and $>50 \%$ reduction in VAS in $82.6 \%$ of participants for an average follow-up time period of $27.8 \pm 4.3$ months. ${ }^{8}$ Zuidema et al also reported significant reduction in VAS scores at 2 and 3 months follow-up (Table 1). ${ }^{7}$

Participants included in studies about groin pain were all aged $\geq 18$ years, diagnosed with chronic groin pain, and have failed previous treatment modalities (Table 2). Participants from case report had also failed treatments with transcutaneous electrical nerve stimulation (TENS) and pulsed radiofrequency prior to successful treatment with DRG stimulation. Detailed parameters of stimulation used in those studies are given in Table 3.

\section{Pelvic girdle pain}

Two case reports with eight participants analyzed effect of neuromodulation with EFS of DRG on pelvic girdle pain., ${ }^{9,10}$ Hunter et al reported significant pain relief in all seven participants after a trial implantation period, whereas four participants reported sustained pain relief 1 year after permanent implantation. ${ }^{9}$ Rowland et al reported a case of $43 \%$ pain reduction from baseline using NRS after 6 months. ${ }^{10}$ Included participants had failed various treatments including medication, neurolysis, surgery, steroid injections, and, in some cases, SCS (Table 2). Details about stimulation parameters are given in Table 3.

\section{Peripheral neuropathy and diabetic peripheral neuropathy}

One case series included ten participants with peripheral diabetic neuropathy who had an average VAS pain reduction from baseline by $64.2 \% \pm 35.8 \%(P<0.001)$ at 12 months. $^{43}$ Participants with peripheral neuropathy were also described in three case reports, of which two reported the same patient, and hence we analyzed only once. ${ }^{29,30,42}$ In both cases, participants reported $>50 \%$ pain relief after 12 months follow-up. Included participants have failed other treatment modalities 
including use of neuropathic pain medications. In van Velsen et $\mathrm{al}^{29}$ and Eldabe et $\mathrm{al}^{43}$ published in 2018, participants also tried traditional SCS, whereas in Maino et $\mathrm{al}^{42}$ TENS was applied prior to neuromodulation with EFS of DRG (Table 2). Only study by Maino et $\mathrm{al}^{42}$ reported small fiber neuropathy diagnosed by skin biopsy, which confirmed pathological reduction of intraepidermal unmyelinated nerve fibers. Other included studies did not clearly document the type of peripheral neuropathy.

\section{Other chronic pain states represented in a single study}

This category includes participants with various painful conditions represented by single case series or case report with less than ten participants. One case report included eight participants with phantom limb pain who were treated with DRG stimulation after a failure of other treatment modalities. The percentage of pain reduction was on average $52 \% \pm 31.9 \%$ from baseline during an average follow-up time period of 9 months. ${ }^{44}$ Other case report included five participants with ACNES. Three participants had good pain relief after 12 months follow-up, whereas two were refractory to the therapy without any pain relief. ${ }^{47}$

Other pain conditions treated with DRG stimulation were chronic intractable pain in the coccyx with $90 \%$ pain reduction at 4 months, ${ }^{45}$ chronic testicular pain with sustained pain reduction of $70 \%-80 \%$ during 1 year, ${ }^{46}$ and LPHS with $>50 \%$ pain relief after 3 years. ${ }^{48}$ These case reports included only one participant.

Details about efficacy and safety of treatment for those indications are given in Table 1, included participants in Table 2 and about parameters of stimulation in Table 3.

\section{Results about safety}

Results about safety of neuromodulation with EFS of DRG could be classified as related to the procedure, related to the device, or related to the stimulation technique. In the group of SAEs related to the procedure, the most common event was infection at the site of implantation. SAEs related to the device included infection of stimulator pocket site, dural puncture, postdural puncture headache, and transient loss of function. The most common SAE related to stimulation was overstimulation. One participant died 6 months after implantation due the medication overdose. This was attributed to previously existing depression. ${ }^{40}$ Several other SAEs occurred that could not be classified as related to implantation procedure or stimulation including depression, bladder infection, bowel obstruction, pain following a capsaicin (Qutenza) application, perianal fistula, knee cyst, transient ischemic attack, worsening of pre-existing CRPS, and temporary loss of leg strength. AEs included loss of stimulation, leads migration, pain at incision site, and postprocedure headache. Incidence of AEs for each study is shown in Table 1.

High proportion of case reports did not report any safety data, ten from included 29 studies. While several studies explicitly mentioned that no complications occurred, it remained unclear if that was true for those studies that did not mention AEs at all.

\section{RoB assessment}

RoB in included studies was assessed using Cochrane RoB tool for the one RCT that was included. We judged domains for random sequence generation and allocation concealment as unclear RoB, as those methods were not reported. Blinding of both participants/personnel and outcome assessors was judged with high RoB because the study was not blinded. The risk of attrition bias was judged as low, since authors reported all attrition during trial, as well as during follow-up period and performed modified intention-to-treat analysis when reporting results. From included 76 participants in both arms, 61 in DRG arm and 54 in SCS arm completed trial period, whereas 12 months follow-up was completed by 55 participants in DRG arm and 50 participants in SCS arm. Reasons for exclusion or failure of treatment were given. We considered that the study had unclear risk of selective reporting bias because in the registered protocol only primary outcome was mentioned and secondary outcomes shown in the manuscript were not mentioned in the protocol. We did not find other sources of bias (Table S4).

\section{Attempts to conduct meta-analysis}

In our study protocol, we planned to conduct a meta-analysis of outcomes reported in RCTs. However, we were unable to conduct a meta-analysis since we found only one RCT. ${ }^{1}$

\section{Reporting of conclusion statements for efficacy and safety in manuscript abstracts}

We were also interested in determining the proportion of studies that reported conclusion statements about efficacy and safety in manuscript abstracts, since sometimes abstracts are the only source of information for clinicians. Such statements for efficacy were either positive conclusive $(\mathrm{N}=12)$ or positive inconclusive $(\mathrm{N}=13)$. The remaining four studies did not report conclusion statements about efficacy (Table S5). 
In the majority of included studies, the abstract did not include any conclusions about the safety of a tested intervention $(\mathrm{N}=19)$. In the remaining abstracts, there were positive conclusive $(\mathrm{N}=5)$ or positive inconclusive statements about safety $(\mathrm{N}=3)$, whereas two abstracts only provided information about number of AEs or mentioned certain specific AEs, without providing overall conclusions about safety (Table S5).

\section{Funding and conflict of interest in included studies}

Most of the included studies had conflict of interest statements. In almost $60 \%$ of the studies, authors reported that they either are consultants of companies providing financial support to the research or that they have equity in those companies. Only nine studies reported that authors have no conflict of interest.

Overall, among 29 studies, there were eight industryfunded studies, only two studies were financed by a nonprofit institution, and none were funded by government or other grant sources. In the group of industry-funded studies, four out of eight had positive conclusive statements about intervention, while four had positive inconclusive statements. Among 21 non-industry funded studies, we found eight studies with positive conclusive statements and nine with positive inconclusive. However, a majority of studies that mentioned a potential conflict of interest did not explicitly mention sources of funding, so we were unable to judge whether those were funded by industry or are they more likely to yield positive findings about intervention.

\section{Discussion}

This systematic review included 29 small studies about the use of EFS of DRG as neuromodulation method for treatment of pain. We found that studies about neuromodulation with EFS of DRG reported participants treated for painful conditions of various etiologies, but mostly in participants who have failed many or all other available treatment modalities. For some participants it was reported that they were refractory to stimulation and that they did not experience any pain relief. The majority of studies that reported conclusion statements about efficacy in their abstracts indicated that there is positive, but inconclusive evidence regarding efficacy of neuromodulation with EFS of DRG. We were unable to perform meta-analysis since only one of the 29 included studies was RCT. ${ }^{27}$

Several reviews have been published recently on this topic, but with a narrower focus and number of methodologi- cal limitations. Harrison et al (2017) published a literature review about efficacy and safety of DRG stimulation as a treatment for neuropathic pain. ${ }^{23}$ Chang Chien et al (2017) published a systematic review about alternate intraspinal targets for SCS. ${ }^{22}$ This review covered very wide range of topics, and DRG stimulation was just one of the analyzed interventions. The review searched only the single database PubMed, whereas the Cochrane Handbook and CRD guidelines indicate that a systematic review requires a search of at least two bibliographic databases. ${ }^{25,27}$ These reviews had also several additional methodological limitations. Specifically, the authors used very simple search strategies, some of them did not report the search dates, they did not report excluded studies, there were no analyses of RoB in included studies, attempts to make quantitative analysis were not reported, and potentially competing interests of authors of included studies were also not reported. None of the studies were focused on participant selection or reported parameters of stimulation.

The latest review published by NACC had some elements of systematic review methodology, including search strategy and analysis of quality of included studies, and gave very comprehensive overview of the topic with sections on DRG anatomy and physiology, with the main focus on DRG stimulation devices and implantation procedure. However, authors mentioned participants' selection very briefly, reported parameters of stimulation only for ACCURATE study and had last date of search in June 2017, which is currently $>1$ year ago. In addition, consensus evidence and given recommendations were partly based on published abstracts without inclusion of full manuscripts. ${ }^{24}$

From all chronic painful conditions treated with neuromodulation with EFS of DRG, CRPS was the condition with evidence represented by ten of the 29 studies included in this systematic review and also with greatest overall number of participants included. Furthermore, this was only condition for which evidence about efficacy and safety was available from RCT. We rated RoB as unclear for multiple domains due to the lack of information provided in manuscript. NACC used modified Pain Physician criteria ${ }^{49}$ and US Preventive Services Task Force (USPSTF) criteria $^{50}$ to give final grading. The group rated ACCURATE study as level 2 according to modified Pain Physician criteria and level I using USPSTF criteria and overall recommended DRG stimulation as an effective therapy for treatment of CRPS type I of the lower extremity, while for upper extremity CRPS type I or II conclusion was that more studies are needed. ${ }^{24}$

NACC had also strong consensus about the use of DRG stimulation for groin pain, rating the overall evidence as 
level II-2. ${ }^{24}$ This is in agreement with our findings since, together with LBP, those were painful conditions represented with uncontrolled before-after studies of higher quality and including more participants than case series and case reports, which reported results for the rest of included chronic painful symptoms. Further studies are needed with higher level evidence about efficacy and safety of neuromodulation with EFS of DRG for treatment of those conditions.

We excluded studies published only as conference abstracts, as it has been shown that such information are not necessarily dependable, as authors may change results, either qualitatively or quantitatively, by the time data from conference abstracts are published in peer-reviewed journals. ${ }^{51}$ Since our search is dated September 2018, we included several painful conditions that have been treated with DRG EFS neuromodulation for the first time such as chronic intractable pain in the coccyx, ${ }^{45} \mathrm{ACNES},{ }^{47}$ and LPHS. ${ }^{48} \mathrm{We}$ also included small pilot studies and case reports that used novel high-frequency parameters of stimulation. ${ }^{5,39}$

This evidence is of low quality, represented only with few participants to whom neuromodulation with EFS of DRG was the last treatment option after failure of other treatment modalities. Median number of participants in these studies was 6 . Based on these findings, future larger studies should also consider inclusion of participants diagnosed with these conditions to confirm safety and efficacy of therapy, as well as use of novel stimulation parameters which could possibly improve treatment outcomes.

\section{Weaknesses of available evidence}

We found that a source of funding was not reported in the majority of included studies. Most of the studies that had financial support were funded by industry with commercial interest in neuromodulation with EFS of DRG, which warrants cautious interpretation of the results. Furthermore, studies that reported potential conflicts of interest in which the authors were either consultants of the industry producing the studied device or had equity in those companies did not report the source of funding. A recently published systematic review about industry sponsorship and research outcome in studies of drugs and medical devices found that industrysponsored research more frequently reported favorable efficacy results and favorable conclusions for tested intervention compared to studies with nonprofit funding. The authors did not find any difference for the majority of RoB items between commercially and nonprofit-funded studies, suggesting that existence of "industry bias" cannot be explained by standard RoB domains. ${ }^{52}$ Amiri et al (2014) reported similar results by analyzing $>1,300$ studies in the field of spine research. They found significant associations between source of funding, study outcome, and level of evidence, in which unfunded and industry-funded studies had the highest proportion of level IV evidence and reported a higher proportion of favorable outcomes, while studies with public funding or funding other than industry had a higher proportion of level I evidence. ${ }^{53}$

Since the source of funding may influence outcomes, reporting sources of funding and conflicts of interest should be a mandatory part of each manuscript. Researchers should follow ethical principles and transparency when reporting study results, while clinicians should critically appraise each paper they are reading, not relying exclusively on the authors' conclusions.

We were aware of the limited number of RCTs in the field as well as the fact that other study designs had lower methodological quality, less reliable results, and thus provide lower level of evidence about certain treatment. ${ }^{27}$ However, NRSDs can be valuable sources of information, having longer follow-up time, especially regarding safety of intervention, which was our outcome of interest, so we decided to include also NRSDs in this systematic review.

Even though we followed criteria for conducting a high-quality systematic review, our evidence synthesis has limitations that are related to the published studies on this topic. The evidence is based on studies with small number of participants, whereas there was only one RCT, and a large proportion of case series and case reports. More reliable evidence is needed to make reliable conclusion about efficacy and safety of studied intervention.

\section{Conclusion}

EFS of DRG is a widely used neuromodulation intervention for treating various painful conditions of different etiologies. Studies published thus far imply that the intervention may help highly selected participants with various pain syndromes, who have failed to achieve adequate pain relief with other pharmacological and nonpharmacological interventions. Some participants were refractory to the treatment, without any pain relief. However, these findings need to be taken with extreme caution because of multiple limitations of available studies. These limitations include poor quality of available studies, very small number of participants included, highly selected patient population who participated in these studies, and conflict of interest of sponsors and authors of those studies. Due to availability of only one trial on this topic, with high or unclear RoB on the majority of analyzed domains, currently available evidence from studies 
on humans about benefits of neuromodulation with EFS of DRG for treatment of pain should be considered preliminary and confirmed in high-quality RCTs with sufficient number of participants.

\section{Acknowledgments}

We are very grateful to Ms Ana Utrobicic, medical librarian from the University of Split School of Medicine, for reviewing the search strategy and helping us to retrieve full texts of manuscripts. This study was supported by the Croatian Science Foundation (HRZZ) grant for Young Scientist Career Development (HRZZ-I-2238-2016) and HRZZ grant for Treating Neuropathic Pain with Dorsal Root Ganglion Stimulation awarded to Prof Damir Sapunar (HRZZ-IP-2013-11-4126).

\section{Disclosure}

The authors report no conflicts of interest in this work.

\section{References}

1. Deer TR, Levy RM, Kramer J, et al. Dorsal root ganglion stimulation yielded higher treatment success rate for complex regional pain syndrome and causalgia at 3 and 12 months: a randomized comparative trial. Pain. 2017;158(4):669-681.

2. Liem L, Russo M, Huygen FJ, et al. One-year outcomes of spinal cord stimulation of the dorsal root ganglion in the treatment of chronic neuropathic pain. Neuromodulation. 2015;18(1):41-49. Discussion 8-9.

3. Liem L, Russo M, Huygen FJ, et al. A multicenter, prospective trial to assess the safety and performance of the spinal modulation dorsal root ganglion neurostimulator system in the treatment of chronic pain. Neuromodulation. 2013;16(5):471-482.

4. Holanda VM, Chavantes MC, Silva DF, et al. Photobiomodulation of the dorsal root ganglion for the treatment of low back pain: a pilot study. Lasers Surg Med. 2016;48(7):653-659.

5. Billet B, Wynendaele R, Vanquathem NE. Wireless neuromodulation for chronic back pain: delivery of high-frequency dorsal root ganglion stimulation by a minimally invasive technique. Case Reports in Medicine. 2017;2017(6):1-4.

6. Huygen F, Liem L, Cusack W, Kramer J. Stimulation of the L2-L3 dorsal root ganglia induces effective pain relief in the low back. Pain Pract. 2018;18(2):205-213.

7. Zuidema X, Breel J, Wille F. Paresthesia mapping: a practical workup for successful implantation of the dorsal root ganglion stimulator in refractory groin pain. Neuromodulation. 2014;17(7):665-669. Discussion 9.

8. Schu S, Gulve A, Eldabe S, et al. Spinal cord stimulation of the dorsal root ganglion for groin pain-a retrospective review. Pain Pract. 2015;15(4):293-299.

9. Hunter CW, Yang A. Dorsal root ganglion stimulation for chronic pelvic pain: a case series and technical report on a novel lead configuration. Neuromodulation. 2019;22(1):87-95.

10. Rowland DC, Wright D, Moir L, Fitzgerald JJ, Green AL. Successful treatment of pelvic girdle pain with dorsal root ganglion stimulation. Br J Neurosurg. 2016;30(6):685-686.

11. Grider JS, Manchikanti L, Carayannopoulos A, et al. Effectiveness of spinal cord stimulation in chronic spinal pain: a systematic review. Pain Physician. 2016;19(1):E33-E54.

12. Pope JE, Deer TR, Kramer J. A systematic review: current and future directions of dorsal root ganglion therapeutics to treat chronic pain. Pain Med. 2013;14(10):1477-1496.
13. Sapunar D, Banozic A, Banozic A, Puljak L. Dorsal root ganglion: a potential new therapeutic target for neuropathic pain. J Pain Res. 2012;5:31-38.

14. Kovalsky Y, Amir R, Devor M. Simulation in sensory neurons reveals a key role for delayed $\mathrm{Na}+$ current in subthreshold oscillations and ectopic discharge: implications for neuropathic pain. $J$ Neurophysiol. 2009;102(3):1430-1442.

15. Koopmeiners AS, Mueller S, Kramer J, Hogan QH. Effect of electrical field stimulation on dorsal root ganglion neuronal function. Neuromodulation. 2013;16(4):304-311.

16. Sapunar D, Ljubkovic M, Lirk P, Mccallum JB, Hogan QH. Distinct membrane effects of spinal nerve ligation on injured and adjacent dorsal root ganglion neurons in rats. Anesthesiology. 2005;103(2):360-376.

17. Gemes G, Koopmeiners A, Rigaud M, et al. Failure of action potential propagation in sensory neurons: mechanisms and loss of afferent filtering in C-type units after painful nerve injury. $J$ Physiol. 2013;591(4):1111-1131.

18. Krames ES. The role of the dorsal root ganglion in the development of neuropathic pain. Pain Med. 2014;15(10):1669-1685.

19. Vuka I, Vučić K, Repić T, Ferhatović Hamzić L, Sapunar D, Puljak L. Electrical stimulation of dorsal root ganglion in the context of pain: a systematic review of in vitro and in vivo animal model studies. Neuromodulation. 2018;21(3):213-224.

20. de Rooij AM, de Mos M, van Hilten JJ, et al. Increased risk of complex regional pain syndrome in siblings of patients? J Pain. 2009;10(12):1250-1255.

21. Albazaz R, Wong YT, Homer-Vanniasinkam S. Complex regional pain syndrome: a review. Ann Vasc Surg. 2008;22(2):297-306.

22. Chang Chien GC, Mekhail N. Alternate intraspinal targets for spinal cord stimulation: a systematic review. Neuromodulation 2017;20(7):629-641.

23. Harrison C, Epton S, Bojanic S, Green AL, Fitzgerald JJ. The efficacy and safety of dorsal root ganglion stimulation as a treatment for neuropathic pain: a literature review. Neuromodulation. 2018;21(3):225-233.

24. Deer TR, Pope JE, Lamer TJ, et al. The neuromodulation appropriateness Consensus Committee on best practices for dorsal root ganglion stimulation. Neuromodulation. 2018;22(1):1-35.

25. Centre for Reviews and Dissemination. Systematic Reviews: CRD's Guidance for Undertaking Reviews in Healthcare. York, UK: University of York; 2009.

26. Moher D, Liberati A, Tetzlaff J, Altman DG, PRISMA Group. Preferred reporting items for systematic reviews and meta-analyses: the PRISMA statement. Open Med. 2009;3(3):e123-e130.

27. Higgins JPT GSe, editor. Cochrane Handbook for Systematic Reviews of Interventions Version 5.1.0 [updated March 2011]. The Cochrane Collaboration; 2011.

28. Sterne JA, Hernán MA, Reeves BC, et al. ROBINS-I: a tool for assessing risk of bias in non-randomised studies of interventions. BMJ. 2016;355:i4919.

29. van Velsen V, van Helmond N, Chapman KB. Creating a strain relief loop during S1 Transforaminal lead placement for dorsal root ganglion stimulation for foot pain: a technical note. Pain Pract. 2018;18(4):539-543.

30. van Velsen V, van Helmond N, Levine ME, Chapman KB. Single-incision approach to implantation of the pulse generator and leads for dorsal root ganglion stimulation: a case report. A A Pract. 2018;10(1):23-27.

31. van Bussel CM, Stronks DL, Huygen F. Dorsal column stimulation vs. dorsal root ganglion stimulation for complex regional pain syndrome confined to the knee: patients' preference following the trial period. Pain Pract. 2018;18(1):87-93.

32. van Bussel CM, Stronks DL, Huygen FJ. Successful treatment of intractable complex regional pain syndrome type I of the knee with dorsal root ganglion stimulation: a case report. Neuromodulation. 2015;18(1):58-60. Discussion 60-61.

33. Yang A, Hunter CW. Dorsal root ganglion stimulation as a salvage treatment for complex regional pain syndrome refractory to dorsal column spinal cord stimulation: a case series. Neuromodulation. 2017;20(7):703-707. 
34. Goebel A, Lewis S, Phillip R, Sharma M. Dorsal root ganglion stimulation for complex regional pain syndrome (CRPS) recurrence after amputation for CRPS, and failure of conventional spinal cord stimulation. Pain Pract. 2018;18(1):104-108.

35. van Buyten J-P, Smet I, Liem L, Russo M, Huygen F. Stimulation of dorsal root ganglia for the management of complex regional pain syndrome: a prospective case series. Pain Pract. 2015;15(3):208-216.

36. Skaribas IM, Peccora C, Skaribas E. Single S1 dorsal root ganglia stimulation for intractable complex regional pain syndrome foot pain after lumbar spine surgery: a case series. Neuromodulation. 2019;22(1):101-107.

37. Weiner RL, Yeung A, Montes Garcia C, Tyler Perryman L, Speck B. Treatment of FBSS low back pain with a novel percutaneous DRG wireless stimulator: pilot and feasibility study. Pain Med. 2016;17(10):1911-1916.

38. Deer TR, Grigsby E, Weiner RL, Wilcosky B, Kramer JM. A prospective study of dorsal root ganglion stimulation for the relief of chronic pain. Neuromodulation. 2013;16(1):67-71.

39. Billet B, Hanssens K, de Coster O, et al. Wireless high-frequency dorsal root ganglion stimulation for chronic low back pain: a pilot study. Acta Anaesthesiol Scand. 2018;62(8):1133-1138.

40. Huygen F, Liem L, Nijhuis H, Cusack W, Kramer J. Evaluating dorsal root ganglion stimulation in a prospective Dutch cohort. Neuromodulation. 2019;22(1):80-86.

41. Morgalla MH, Fortunato M, Lepski G, Chander BS. Dorsal root ganglion stimulation (DRGs) for the treatment of chronic neuropathic pain: a single-center study with long-term prospective results in 62 cases. Pain Physician. 2018;21(4):E377-E87.

42. Maino P, Koetsier E, Kaelin-Lang A, Gobbi C, Perez R. Efficacious dorsal root ganglion stimulation for painful small fiber neuropathy: a case report. Pain Physician. 2017;20(3):E459-E63.

43. Eldabe S, Espinet A, Wahlstedt A, et al. Retrospective case series on the treatment of painful diabetic peripheral neuropathy with dorsal root ganglion stimulation. Neuromodulation. 2018;21(8):787-792.
44. Eldabe S, Burger K, Moser H, et al. Dorsal root ganglion (DRG) stimulation in the treatment of phantom limb pain (PLP). Neuromodulation. 2015;18(7):610-617.

45. Giordano NL, van Helmond N, Chapman KB. Coccydynia treated with dorsal root ganglion stimulation. Case Rep Anesthesiol. 2018;2018(1):1-4.

46. Hassanain M, Murphy P. Dorsal root ganglion stimulation for the treatment of bilateral intractable chronic testicular pain. Neuromodulation. 2019;22(1):115-116.

47. Mol FMU, Roumen RMH. DRG spinal cord stimulation as solution for patients with severe pain due to anterior cutaneous nerve entrapment syndrome: a case series. Neuromodulation. 2018;21(3):317-319.

48. Zuidema X, Schapendonk J. Dorsal root ganglion stimulation: a treatment option for chronic pain due to refractory loin pain haematuria syndrome. Neuromodulation. 2017;20(8):841-843.

49. Manchikanti L, Hirsch JA, Cohen SP, et al. Assessment of methodologic quality of randomized trials of interventional techniques: development of an interventional pain management specific instrument. Pain Physician. 2014;17(3):E263-290.

50. Harris RP, Helfand M, Woolf SH, et al. Current methods of the US Preventive Services Task force: a review of the process. Am J Prev Med. 2001;20(3 Suppl):21-35.

51. Saric L, Vucic K, Dragicevic K, et al. Comparison of conference Abstracts and full-text publications of randomized controlled trials presented at four consecutive world Congresses of pain: reporting quality and agreement of results. Eur J Pain. 2019;23(1):107-116.

52. Lundh A, Lexchin J, Mintzes B, Schroll JB, Bero L. Industry sponsorship and research outcome. Cochrane Database Syst Rev. 2017;2:MR000033.

53. Amiri AR, Kanesalingam K, Cro S, Casey AT. Does source of funding and conflict of interest influence the outcome and quality of spinal research? Spine J. 2014;14(2):308-314.
Journal of Pain Research

\section{Publish your work in this journal}

The Journal of Pain Research is an international, peer reviewed, open access, online journal that welcomes laboratory and clinical findings in the fields of pain research and the prevention and management of pain. Original research, reviews, symposium reports, hypothesis formation and commentaries are all considered for publication.

\section{Dovepress}

The manuscript management system is completely online and includes a very quick and fair peer-review system, which is all easy to use. Visit http://www.dovepress.com/testimonials.php to read real quotes from published authors. 